\title{
Coherent X-ray imaging across length scales
}

\author{
Peter R.T. Munro *
}

\begin{abstract}
Contemporary X-ray imaging techniques span a uniquely wide range of spatial resolutions, covering five orders of magnitude. The evolution of X-ray sources, from the earliest laboratory sources through to highly brilliant and coherent free electron lasers, has been key to the development of these imaging techniques. This review surveys the predominant coherent X-ray imaging techniques with fields of view ranging from that of entire biological organs, down to that of biomolecules. We introduce the fundamental principles necessary to understand the image formation for each technique as well as briefly reviewing coherent X-ray source development. We present example images acquired using a selection of techniques, by leaders in the field.
\end{abstract}

\section{Introduction}

X-rays have been extremely important to a very broad range of research fields since their discovery by Wilhelm Röntgen in 1895 [1]. The importance of this discovery is not exaggerated by Röntgen receiving the very first Nobel prize for physics, in 1901, as a consequence. The X-rays generated by Röntgen were far from coherent when compared to those generated by sources available today. The contrast between Röntgen's source and a free electron laser is similar to that between a low power incandescent globe and an optical laser. Just as the laser enabled a revolution in optical imaging, so too have coherent X-ray sources.

X-ray imaging modalities possess some important strengths relative to optical imaging modalities. One strength is that X-rays can propagate deep within matter, which is why Xray imaging is so widely used in medicine. Imaging on this macroscopic scale has, arguably, been undergoing its most significant advance since the invention of computed tomography, due to the advent of phase contrast imaging. Phase contrast imaging generates contrast based on the speed of X-rays within matter, rather than their attenuation. Phase contrast

*Department of Medical Physics and Biomedical Engineering, University College London, WC1E 6BT, UK and the School of Electrical, Electronic and Computer Engineering, The University of Western Australia, Crawley, WA 6009, Australia (e-mail: p.munro@ucl.ac.uk) 
imaging, which has proven to be a very effective tool for imaging soft biological tissue, became prominent only relatively recently when the third-generation synchrotrons, which produce highly coherent X-ray radiation, were developed $[2,3]$. Another strength of X-rays is that their short wavelength means that diffraction limited X-ray imaging techniques can be applied at a spatial resolution sufficient to perform molecular scale imaging [4]. Such imaging is, however, completely reliant upon highly coherent and brilliant free electron laser X-ray sources, of which only four currently exist throughout the world.

X-rays can thus be applied to imaging at spatial resolutions covering a broad range of length scales. They have been, and continue to be, be a vital tool in biological research, since they can be used to image at the organ, tissue, cell and molecular scales. This tutorial review surveys the imaging techniques which have been developed to image at each of these scales. We commence by briefly reviewing the production of X-rays and their interaction with matter. We then consider what makes an X-ray source coherent and survey the evolution of such sources. The principal coherent X-ray imaging techniques spanning the macroscopic, microscopic and molecular length scales are considered, along with example images from several of the modalities. Owing to the tutorial nature of this review, a degree of rigour has been omitted for the benefit of clarity. Further, a great many works of significance have not been cited due to the breadth of techniques considered in this review. Readers interested in more comprehensive reviews of particular aspects covered in this review are directed to a number of excellent reviews [5-13].

\section{Properties of coherent X-rays and their production}

\subsection{Interaction of coherent X-rays with matter}

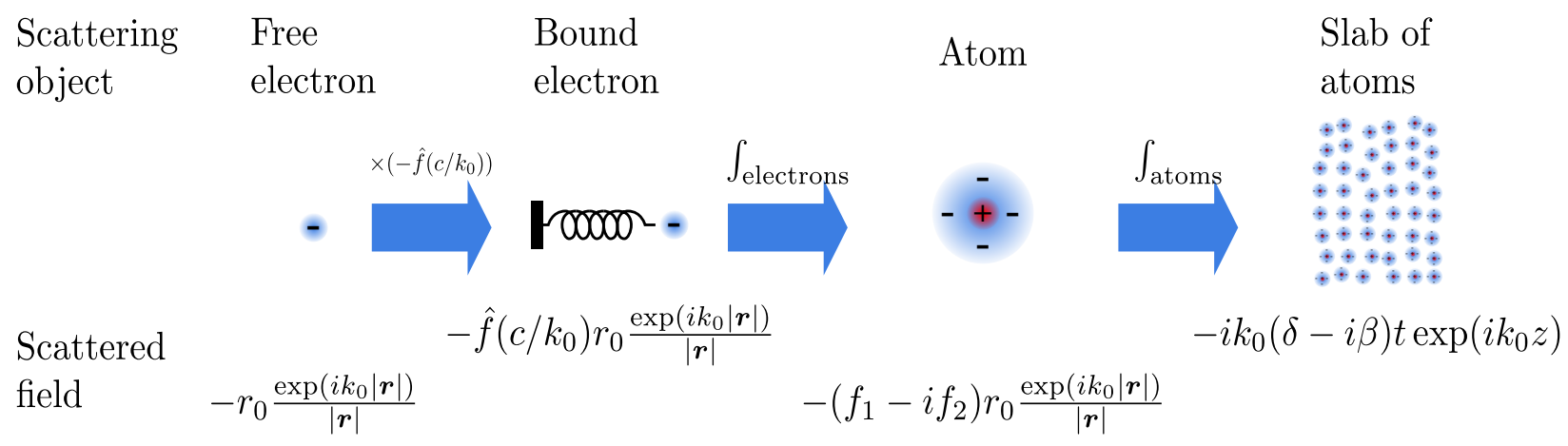

Figure 1: Illustration of how the field scattered by a slab of atoms can be related to the field scattered by a single, free electron. The equation of the scattered field, for each scenario, is given along the bottom of the figure.

Understanding the interaction of coherent X-rays with matter is a key requirement for understanding image formation in all coherent X-ray imaging modalities. Since we are interested 
in coherent imaging, we neglect inelastic scattering, whereby scattered X-rays differ in energy from unscattered X-rays. Our ultimate objective is to describe how X-rays are scattered by matter on both macroscopic and molecular length scales. The aim of this section is to relate the scattering properties of a single, free, electron to that of bulk material. The sequence of steps required to make this link is illustrated in Fig. 1, beginning with the field scattered by a single, free electron, for an incident plane wave. Each of the scenarios depicted in Fig. 1 is explained in detail in this section. By way of introduction, the field scattered by a bound electron is found by multiplying that of a free electron field by a frequency dependent term $\hat{f}\left(c / k_{0}\right)$. This frequency dependent term takes account of resonance and damping phenomena experienced by bound electrons. The field scattered by an atom is found by summing the fields due to each bound electron contained within it. Finally, the field scattered by bulk matter can be obtained by summing up the field scattered by ensembles of atoms, at which point the concept of refractive index emerges. We shall now consider this progression in further detail

We begin by considering scattering by a single electron, at the origin, as shown in Fig. 2. At this stage we do not make any assumptions about whether the electron is free or bound. We assume that a linearly polarised plane wave of the form $\boldsymbol{E}_{i n c}(z, t)=E_{0} \hat{\boldsymbol{e}}_{\text {inc }} \exp \left(i\left(k_{0} z-\omega t\right)\right)$ is incident upon the electron, where $\hat{\boldsymbol{e}}_{i n c}$ is the direction of polarisation, $\omega$ is the angular frequency of oscillation, $E_{0}$ is the wave amplitude and $k_{0}$ is the wave number. We denote the position of the electron by $\boldsymbol{x}(t)$, and so when the electron, having charge $e$ and rest mass $m_{e}$, experiences an acceleration, $\ddot{\boldsymbol{x}}(t)$, due to the incident electric field, the electron will radiate an electric field, at position $\boldsymbol{r}=|\boldsymbol{r}| \hat{\boldsymbol{r}}$ as [14]

$$
\boldsymbol{E}_{a}(\boldsymbol{r}, t)=\frac{e}{4 \pi \epsilon_{0} c^{2}} \frac{\hat{\boldsymbol{r}} \times(\hat{\boldsymbol{r}} \times \ddot{\boldsymbol{x}}(t-|\boldsymbol{r}| / c))}{|\boldsymbol{r}|} .
$$

If the electron is free, its acceleration will be determined purely by the incident electric field as

$$
\ddot{\boldsymbol{x}}(t)=\frac{-e}{m_{e}} \boldsymbol{E}_{\text {inc }}(0, t) .
$$

Electrons are, however, generally bound and can be treated as damped oscillating dipoles for incident electric field photon energies away from resonant energies. An electron can be ejected from the atom at resonant energies and a different treatment is required. Away from resonant energies, the equation of motion of the electron takes the form

$$
\ddot{\boldsymbol{x}}(t)+\gamma_{i} \dot{\boldsymbol{x}}(t)+\omega_{i}^{2} \boldsymbol{x}(t)=\frac{-e}{m_{e}} \boldsymbol{E}_{\text {inc }}(0, t)
$$

where $\gamma_{i}$ is a damping factor and $\omega_{i}$ is the electron's resonant frequency. By assuming that $\boldsymbol{x}(t)$ undergoes harmonic oscillation with frequency $\omega$, we can write Eq. (3) as

$$
\begin{aligned}
\ddot{\boldsymbol{x}}(t) & =\frac{\omega^{2}}{\omega^{2}-\omega_{i}^{2}+i \gamma_{i} \omega} \frac{-e}{m_{e}} \boldsymbol{E}_{i n c}(0, t) \\
& =\left(\hat{f}_{1}(\omega)-i \hat{f}_{2}(\omega)\right) \frac{-e}{m_{e}} \boldsymbol{E}_{i n c}(0, t)
\end{aligned}
$$


where we introduce $\hat{f}(\omega)=\hat{f}_{1}(\omega)-i \hat{f}_{2}(\omega)$ as the factor which multiplies the scattered field of a free electron to give that due to a bound electron. Eq. (5) can then be substituted into Eq. (1) to calculate the field radiated by the bound electron.

We note that the expression in Eq. (1) depends strongly upon the polarisation direction of $\boldsymbol{E}_{\text {inc }}$ relative to the point of observation, $\boldsymbol{r}$. If $\hat{\boldsymbol{r}}$ makes only a small angle with the $z$-axis and if unpolarised X-rays are used, Eqs. (1) and (5) may be combined and simplified to yield the complex amplitude irradiated by a bound electron as $[14,15]$

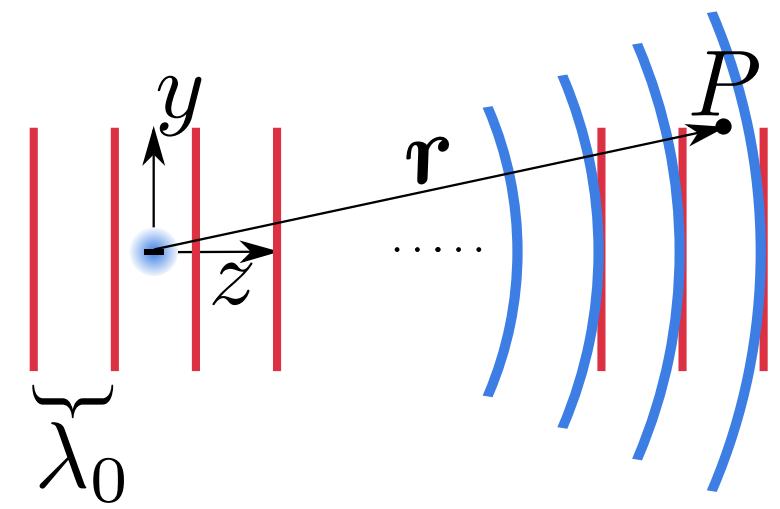

Figure 2: Diffraction of an incident plane wave with wavelength $\lambda_{0}$, by a free electron located at the origin of our coordinate system. We observe the diffracted field at position $P$ which is displaced by $\boldsymbol{r}$ relative to the electron. $P$ is assumed to be a long way from the electron.

$$
\psi(\boldsymbol{r}) \sim \exp \left(i k_{0} \boldsymbol{r} \cdot \hat{\boldsymbol{k}}\right)-\hat{f}\left(c / k_{0}\right) r_{0} \frac{\exp \left(i k_{0}|\boldsymbol{r}|\right)}{|\boldsymbol{r}|}
$$

where

$$
r_{0}=\frac{e^{2}}{4 \pi \epsilon_{0} m_{e} c^{2}}
$$

is the classical electron radius and $\hat{\boldsymbol{k}}$ is the unit vector parallel to the $z$-axis. The first term in Eq. (6) represents the incident electric field and the second term represents the scattered field. The representation of the two fields in this way is called the first Born approximation $[16]$.

We are, however, interested in scattering by atoms and collections of atoms rather than single electrons. If we consider an electron density distribution, $\rho(\boldsymbol{x})$, then the amplitude of $\mathrm{X}$-rays scattered by this distribution of electrons can be found by integrating the scattered component of Eq. (6) over $\rho(\boldsymbol{x})$, and accounting for the phase variation in the incident wave resulting from the electron displacement, i.e., 


$$
\begin{aligned}
\Psi(\boldsymbol{r}) & =-\int_{-\infty}^{\infty} \hat{f}\left(c / k_{0}\right) r_{0} \frac{\exp \left(i k_{0}|\boldsymbol{r}-\boldsymbol{x}|\right)}{|\boldsymbol{r}-\boldsymbol{x}|} \exp \left(i k_{0} \hat{\boldsymbol{k}} \cdot \boldsymbol{x}\right) \rho(\boldsymbol{x}) \mathrm{d}^{3} \boldsymbol{x} \\
& \approx-\hat{f}\left(c / k_{0}\right) r_{0} \frac{\exp \left(i k_{0}|\boldsymbol{r}|\right)}{|\boldsymbol{r}|} \int_{-\infty}^{\infty} \exp \left(-i k_{0} \hat{\boldsymbol{r}} \cdot \boldsymbol{x}\right) \exp \left(i k_{0} \hat{\boldsymbol{k}} \cdot \boldsymbol{x}\right) \rho(\boldsymbol{x}) \mathrm{d}^{3} \boldsymbol{x}
\end{aligned}
$$

We note that for atoms, $\hat{f}\left(c / k_{0}\right)$ is obtained by summing Eq. (4) over all electron resonances, $\omega_{i}$, and associated damping factors, $\gamma_{i}$. Equation (8) can be used to analyse many X-ray scattering applications. For example, if $\rho(\boldsymbol{x})$ is a function periodic in $\boldsymbol{x}$, such as in Xray crystallography, the scattered X-rays will form an intensity pattern with periodic sharp maxima. If $\rho(\boldsymbol{x})=\rho_{a}(\boldsymbol{x})$ represents the electron distribution owing to a single atom, Eq. (8) is written as

$$
\begin{aligned}
& \Psi_{a}(\boldsymbol{r}) \\
& \quad=-\hat{f}\left(c / k_{0}\right) r_{0} \frac{\exp \left(i k_{0}|\boldsymbol{r}|\right)}{|\boldsymbol{r}|} \int_{-\infty}^{\infty} \exp \left(-i k_{0} \hat{\boldsymbol{r}} \cdot \boldsymbol{x}\right) \exp \left(i k_{0} \hat{\boldsymbol{k}} \cdot \boldsymbol{x}\right) \rho_{a}(\boldsymbol{x}) \mathrm{d}^{3} \boldsymbol{x} \\
& \quad=-\left(f_{1}-i f_{2}\right) r_{0} \frac{\exp \left(i k_{0}|\boldsymbol{r}|\right)}{|\boldsymbol{r}|}
\end{aligned}
$$

where $f=f_{1}-i f_{2}$, implicitly defined, is the atomic scattering factor which takes into account the bound nature of the electrons as well as their spatial distributions and have been tabulated by Henke et al. [17]. We can use the result in Eq. (10) to non-rigorously derive an expression for the refractive index at X-ray energies, usually written as $n=1-\delta+i \beta$. Assume, to begin with, that we have a random arrangement of atoms within a slab extending from $z=0$ to $z=t$, having a volume density of $n_{a}$ atoms per unit volume. We assume, as in Eq. (6), that we have a plane wave normally incident upon the slab of atoms. The total scattered field at some location downstream of the slab is given by integrating over Eq. (10) as

$$
\Psi_{s}(\boldsymbol{r})=\int_{-\infty}^{\infty} \int_{0}^{t} n_{a} \Psi_{a}(\boldsymbol{r}-\boldsymbol{x}) \exp \left(i k_{0} \hat{\boldsymbol{k}} \cdot \boldsymbol{x}\right) \mathrm{d} z \mathrm{~d}^{2} \boldsymbol{x}_{\perp} .
$$

Equation (11) can be understood intuitively by noting that the incident plane wave has a complex amplitude of $\exp \left(i k_{0} \hat{\boldsymbol{k}} \cdot \boldsymbol{x}\right)$ at location $\boldsymbol{x}$. If an atom is located at position $\boldsymbol{x}$, instead of the origin, the scattered field propagates a distance $\boldsymbol{r}-\boldsymbol{x}$ to $\boldsymbol{r}$, the point of observation. The scattered field due to a distribution of atoms can thus be found by summing all of the scattered field components originating from within the slab. We can use Eq. (11) to evaluate the field scattered by an object made of a single material and a variable thickness. We start by defining the point of observation, $\boldsymbol{r}=\left(\boldsymbol{r}_{\perp}, z\right)$ where $\boldsymbol{r}_{\perp}$ represents the position in the 


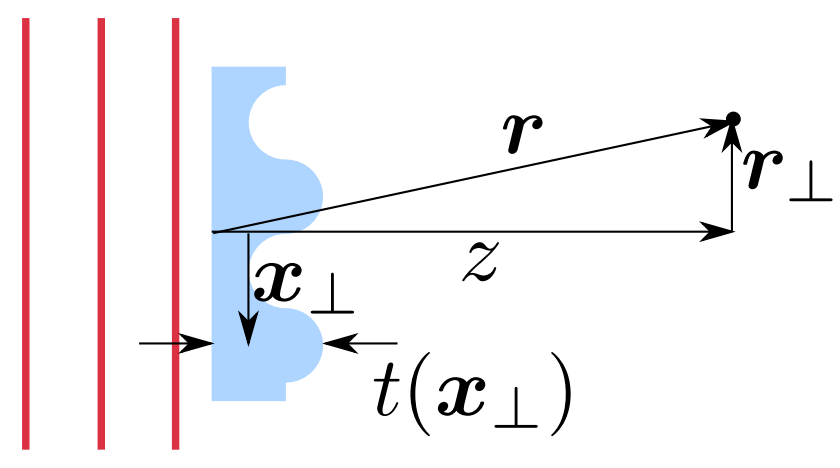

Figure 3: An illustration of the coordinate system used to formulate the Kirchhoff-Fresnel diffraction integral in Eq. (11).

$x y$ plane as shown in Fig. 3. We similarly define $\boldsymbol{x}_{\perp}$ as the transverse position within the sample and thus define $t\left(\boldsymbol{x}_{\perp}\right)$ as the thickness of the sample at transverse position $\boldsymbol{x}_{\perp}$. By defining the refractive index at X-ray energies as $n=1-\delta+i \beta$, it is possible to calculate the total field which arises downstream of the object (see Appendix A) as

$$
\begin{aligned}
\Psi_{t}(\boldsymbol{r}) & =\exp \left(i k_{0} z\right)+\Psi_{s}(\boldsymbol{r}) \\
& =\frac{\exp \left(i k_{0} z\right)}{i \lambda z} \int_{-\infty}^{\infty} \exp \left(k_{0}(-i \delta-\beta) t\left(\boldsymbol{x}_{\perp}\right)\right) \exp \left(i k_{0} \frac{\left|\boldsymbol{r}_{\perp}-\boldsymbol{x}_{\perp}\right|^{2}}{2 z}\right) \mathrm{d}^{2} \boldsymbol{x}_{\perp} .
\end{aligned}
$$

Equation 12 is recognisable as being equivalent to the Kirchhoff-Fresnel diffraction integral [18] widely used in optics. Another notable feature of Eq. (12) is that a three-dimensional object is represented as a two-dimensional transparency function, $\exp \left(k_{0}(-i \delta-\beta) t\left(\boldsymbol{x}_{\perp}\right)\right)$. This approach is known as the projection approximation [15] and is valid for samples where, from a ray optics point of view, rays emerge from the sample at approximately the same place they would have in the absence of the sample, albeit with a possibly altered direction of propagation. A more rigorous analysis can be used to show that the projection approximation requires that $t\left(\boldsymbol{x}_{\perp}\right)$ does not vary "too quickly". It has, however, been shown that the projection approximation is a very good one for application in coherent X-ray imaging [19].

\subsection{Coherence properties}

Coherent X-ray imaging experiments are normally designed such that we can assume a wavefield with complete temporal coherence, which is equivalent to saying that the wavefield is quasi-monochromatic. The same cannot be said for spatial coherence and we derive some of the fundamental concepts used to describe spatial coherence. We start by considering Young's interference experiment, a thorough account of which is given by Wolf [20]. We are ultimately interested in calculating the radiance of the field at a location $\boldsymbol{r}_{o}$, some distance downstream of an opaque screen with two pinholes at positions $\boldsymbol{r}_{1}$ and $\boldsymbol{r}_{2}$. We take the 
origin of our coordinate system to be the midpoint between the two pinholes. The scenario under consideration is depicted in Fig. 4. We begin by considering the field due to just one of the pinholes, say the one at $\boldsymbol{r}_{1}$. If we consider Eq. (12) where the screen is represented by a slab with a large $\beta$ value and a thickness $t\left(\boldsymbol{x}_{\perp}\right)$, which is non-zero everywhere except the pinhole, the field at $\boldsymbol{r}_{o}$ due to the pinhole at $\boldsymbol{r}_{1}$ is given by

$$
\Psi\left(\boldsymbol{r}_{o}, t ; \boldsymbol{r}_{1}\right)=\Psi\left(\boldsymbol{r}_{1}\right) \exp (-i \omega t) \exp \left(i k_{0} t_{1} c\right) /\left(i \lambda t_{1} c\right)
$$

where we have substituted $t_{1} c=\left|\boldsymbol{r}_{o}-\boldsymbol{r}_{1}\right|$ and $c$ is the speed of light in a vacuum and $\Psi\left(\boldsymbol{r}_{1}\right) \exp (-i \omega t)$ is the field incident upon the pinhole. The observed intensity due to any wavefield will always be averaged over many temporal fluctuations of the wavefield, which is assumed to be stationary. Considering a fluctuating field $\Psi(\boldsymbol{r}, t)$, the instantaneous intensity is given by $\Psi^{*}(\boldsymbol{r}, t) \Psi(\boldsymbol{r}, t)$, where ${ }^{*}$ represents complex conjugation. The observed intensity, which we denote $I(\boldsymbol{r})$ is given by

$$
I(\boldsymbol{r})=\lim _{T \rightarrow \infty} \frac{1}{T} \int_{0}^{T} \Psi^{*}(\boldsymbol{r}, t) \Psi(\boldsymbol{r}, t) \mathrm{d} t .
$$

Considering now the wavefield due to the two pinholes in Fig. 4, the observed intensity at $\boldsymbol{r}_{o}$ is found by substituting $\Psi\left(\boldsymbol{r}_{o}, t\right)=\Psi\left(\boldsymbol{r}_{o}, t ; \boldsymbol{r}_{1}\right)+\Psi\left(\boldsymbol{r}_{o}, t ; \boldsymbol{r}_{2}\right)$ into Eq. (14). By defining $\omega_{0}=c k_{0}, K_{1}=1 /\left(i \lambda t_{1} c\right)$ and $K_{2}=1 /\left(i \lambda t_{2} c\right)$ it is straightforward to show that

$$
I\left(\boldsymbol{r}_{o}\right)=\left|K_{1}\right|^{2} I\left(\boldsymbol{r}_{1}\right)+\left|K_{2}\right|^{2} I\left(\boldsymbol{r}_{2}\right)+2 \Re\left\{\left|K_{1}\right|\left|K_{2}\right| \Gamma\left(\boldsymbol{r}_{1}, \boldsymbol{r}_{2}, t_{1}-t_{2}\right)\right\},
$$

where $\Re$ denotes the real part and $\Gamma\left(\boldsymbol{r}_{1}, \boldsymbol{r}_{2}, \tau\right)=\Psi^{*}\left(\boldsymbol{r}_{1}\right) \Psi\left(\boldsymbol{r}_{2}\right) \exp \left(-i \omega_{0} \tau\right)$ is the mutual coherence function for the special case of quasi-monochromatic radiation. The cross-spectral density function is defined as the Fourier transform of the mutual coherence function, which, in the special case of quasi-monochromatic radiation is given by

$$
W\left(\boldsymbol{r}_{1}, \boldsymbol{r}_{2}, \omega\right)=\int_{-\infty}^{\infty} \Gamma\left(\boldsymbol{r}_{1}, \boldsymbol{r}_{2}, \tau\right) \exp (i \omega \tau) \mathrm{d} \tau=J\left(\boldsymbol{r}_{1}, \boldsymbol{r}_{2}\right) \delta\left(\omega-\omega_{0}\right)
$$

where $\delta(x)$ is Dirac's delta function and $J\left(\boldsymbol{r}_{1}, \boldsymbol{r}_{2}\right)$ is the mutual optical intensity function. We can now state a concept which is very important to both mathematical methods in coherent X-ray imaging and intuitive understanding. It can be shown rigorously that the mutual optical intensity function can be expressed, in the quasi-monochromatic case, as superposition of coherent modes, i.e. [21]

$$
J\left(\boldsymbol{r}_{1}, \boldsymbol{r}_{2}\right)=\sum_{n} \alpha_{n} \phi_{n}^{*}\left(\boldsymbol{r}_{1}\right) \phi_{n}\left(\boldsymbol{r}_{2}\right)
$$

Each of the modes, $\phi_{n}(\boldsymbol{r})$, are mutually incoherent and the mode occupancy, $\alpha_{n}$, indicates the dominance of the mode. The measured quantity, intensity, is given by Eq. (17) as

$$
I(\boldsymbol{r})=\sum_{n} \alpha_{n} \phi_{n}^{*}(\boldsymbol{r}) \phi_{n}(\boldsymbol{r})=\sum_{n} \alpha_{n} I_{n}(\boldsymbol{r})
$$


where $I_{n}$ is the intensity of the $n$th mode. Note that this mathematically expresses the concept that when partially spatially coherent wavefields are used, the total intensity is formed by the sum of intensities corresponding to each of a set of completely spatially coherent modes. The modes cannot be chosen arbitrarily, but are the eigenfunctions of an integral equation [21]. As an example, however, the modes due to an electron bombardment source have the form $\phi_{n}(\boldsymbol{r})=\left(1 /\left|\boldsymbol{r}-\boldsymbol{x}_{n}\right|\right) \exp \left(i k\left|\boldsymbol{r}-\boldsymbol{x}_{n}\right|\right)$, where each $\boldsymbol{x}_{n}$ represents a position on the source's electron target. A wavefield with complete spatial coherence has a single mode in the summation of Eq. (18). Each of the modes in Eq. (17) can be propagated between two planes in free space using Eq. (12), assuming that the slab thickness is set to 0 everywhere, and by including the mode within the kernel of the integral. The modes can also be propagated through a scattering object using the same approach using an arbitrary thickness function. Supposing then that after propagation, either through homogeneous or inhomogeneous space, a mode is transformed from $\phi_{n}(\boldsymbol{r})$ to $\Phi_{n}(\boldsymbol{r})$, the intensity of the propagated field is given by

$$
I(\boldsymbol{r})=\sum_{n} \alpha_{n} \Phi_{n}^{*}(\boldsymbol{r}) \Phi_{n}(\boldsymbol{r})
$$

meaning that all of the techniques which have been developed for propagating coherent wavefields can also be employed for partially spatially coherent wavefields.

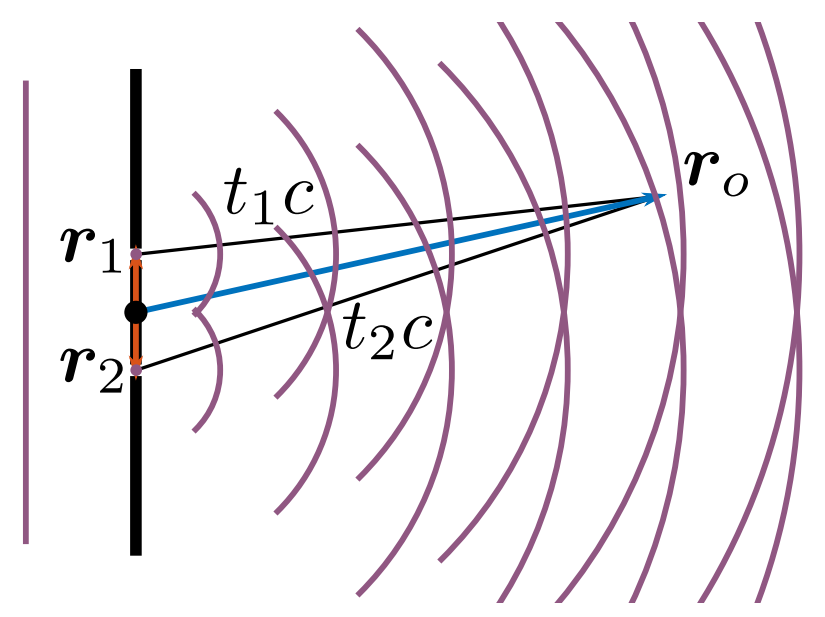

Figure 4: A plane wave incident upon a screen with pinholes at locations $\boldsymbol{r}_{1}$ and $\boldsymbol{r}_{2}$.

\subsection{Evolution of coherent X-ray sources}

The evolution of coherent X-ray sources has been integral to the development of the field of coherent X-ray imaging. This is underlined by Fig. 7 which shows how source brilliance (which will be defined later) has increased by 20 orders of magnitude over six decades. The progression continues to facilitate the development of new imaging techniques. The earliest $\mathrm{X}$-ray source generated electrons by bombarding relativistic electrons into a metal target known as an anode. Indeed, this method was used by Röntgen when he discovered X-ray 
radiation [1]. This type of source, which is the mainstay of medical X-ray imaging, creates radiation by two phenomena. One phenomenon is referred to as "bremsstrahlung", a German word which translates to "braking radiation" [15]. As its name suggests, this radiation is generated as a result of the rapid deceleration of relativistic electrons in the anode. The spectrum of emitted photon energies is very broad, spanning from very low energies up to the peak kinetic energy of incident electrons. This kind of radiation is therefore temporally incoherent. The second phenomenon by which X-rays are produced leads to sharp peaks in the spectrum, superimposed on the bremsstrahlung spectrum. These peaks, known as "characteristic lines", result from bound electrons being ejected from atoms at particular energies as discussed in Sec. (2.1). When this occurs, electrons residing at higher energy levels fill the vacancy left by the ejected electron, leading to the emission of a photon. As will be briefly discussed later, characteristic lines can be used to generate a source which is close to quasi-monochromatic.

We earlier alluded to the term brilliance, which is an important definition for coherent Xray sources. Brilliance is defined as the number of photons within a narrow energy band, normalised by the transverse area of the beam and its angular divergence. The energy bandwidth is usually $0.1 \%$ of the central photon energy. The typical units of brilliance are thus photons/(sec $\left.\mathrm{mm}^{2} \operatorname{mrad}^{2} 0.1 \% \mathrm{BW}\right)$.

The brilliance of any beam of radiation cannot be changed once it has been produced [22]. This can be understood intuitively from Fig. 6 which shows examples of two different beams being focused. The idealised beam to the left of the lens in Fig. 6a) has a very small angular divergence, meaning that the beam must have a very broad diameter. As a result, this broad, low divergence, beam may be focused to a diffraction limited spot with angular divergence $\theta_{1}$. The focused beam has a large divergence angle and narrow focal spot, compared with the small divergence angle and broad beam diameter of the unfocused beam. This qualitatively demonstrates that the product of angular divergence and beam diameter is conserved. In contrast, the beam to the left of the lens in Fig. 6b) has an angular divergence $\theta_{0}$ and is focused to a beam having the same angular divergence as in a), but with a broader focal spot. So if the beams in a) and b) had an equal number of photons, the beam in a) would have a higher brilliance, since its product of beam diameter and beam divergence is smaller than that of $b$ ). This is further demonstrated by the focused beam in Fig. 6a) being narrower than Fig. 6b), meaning that a greater number of photons must be in the focal region of Fig. $6 a)$.

Heat generation within the anode is the main factor which limits the brilliance achievable by electron bombardment sources. A typical source employing a tungsten anode, accelerating electrons to $140 \mathrm{keV}$, is only approximately $1 \%$ efficient, meaning that $99 \%$ of the electrons' energy is converted into heat within the anode [23]. Brilliance has been increased by developing rotating anodes with advanced cooling systems, thus allowing more electrons to bombard the anode, per unit of time. Brilliance has also been increased by tightly focusing the electron beam which bombards the anode. 
Despite the improvement in electron bombardment sources, often termed "conventional" sources, synchrotrons have led the way in terms of source brilliance as demonstrated in Fig. 5. It is important to note that Fig. 5 represents a trend which broadly fits data obtained from several sources [24-27]. Synchrotrons have been responsible for a significant increase in source brilliance. Synchrotron radiation originates from the acceleration of relativistic charged particles along a curved trajectory, and is thus quite different in nature from that of conventional sources. Three generations of synchrotrons can be identified as indicated in Fig. 5. The first generation of synchrotrons produced X-ray radiation as a by-product rather than by design. Radiation was produced by particle accelerators intended for high energy particle physics experiments and was considered undesirable in such instruments, since it led to energy loss. Second-generation synchrotrons were specifically designed to produce X-ray radiation. The main development in going from first- to second-generation synchrotrons was the advent of storage rings, the purpose of which was to maintain electrons in a circular orbit at fixed energy. The Synchrotron Radiation Source (SRS) at the Daresbury Laboratory in the UK was the first of the second-generation of synchrotrons, which became operational in 1981. X-ray radiation is generated in second-generation synchrotrons by the use of bending magnets which produce a magnetic field within the storage ring, directed normal to both the trajectory of the electrons and lines joining the location of the magnet to the centre of the storage ring. The bending magnets are essential, not just for producing X-ray radiation, but also for maintaining the electron orbit within the storage ring, which is not strictly a ring, but a series of straight segments joined together by bending magnets.

The third generation of synchrotrons achieved a further increase in brilliance by generating $\mathrm{X}$-ray radiation from within the straight segments of the storage ring. This was achieved by the use of so-called "wigglers" and "undulators" being inserted into the straight segments. The devices employ magnetic fields with alternating polarity, as shown in Fig. 7, which cause the electron beam to propagate along an approximately sinusoidal trajectory. The next generation of light sources, so-called fourth-generation sources, are free electron lasers. As with optical lasers in the longer wavelengths, free electron lasers make use of stimulated emission, yet they do not employ a pump medium. Instead, free electron lasers employ an undulator which is sufficiently long such that X-ray photons produced by stimulated emission are dominant compared with those produced by spontaneous emission. In particular, at a certain point along the undulator, the electrons experience an electromagnetic field due to the undulator and X-ray photons. Under the conditions of the free electron laser, the interaction of electrons and the X-ray photons is strong enough to stimulate the emission of further X-ray photons which are coherent with the previously existing X-ray photons. Free electron lasers are capable of producing beams of very high brilliance which are currently enabling new basic science to be performed [4]. 


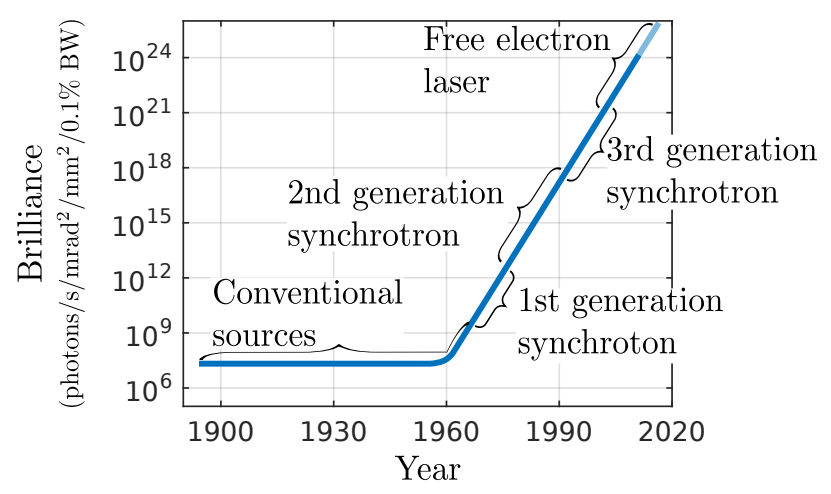

Figure 5: An indicative evolution of X-ray source brilliance since X-rays were first detected, showing how brilliance has increased by 20 orders of magnitude over a period of six decades [24-27].
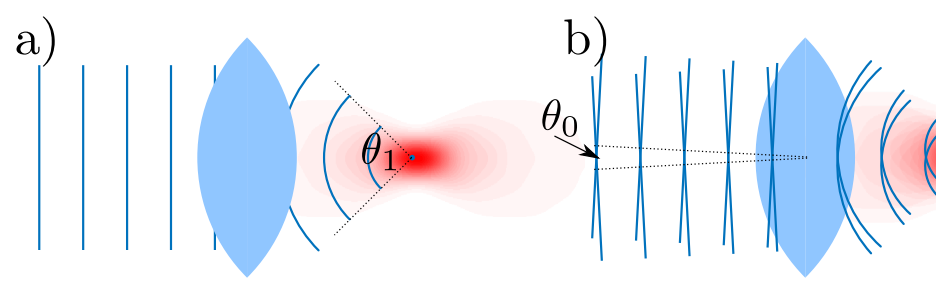

Figure 6: The brilliance of a beam cannot be increased by focusing optics. A beam with little divergence can be focused to a diffraction limited spot (as in a) and angular divergence $\theta_{1}$. A beam with angular divergence $\theta_{0}$ may be focused to a wider focal spot and angular divergence $\theta_{1}$ (as in b).

\section{Coherent X-ray imaging techniques}

Having covered the necessary preliminary material, we can now discuss the coherent Xray imaging techniques which have been developed to acquire images across the spectrum of length scales which covers five orders of magnitude. Source development has been integral in enabling the development of these techniques, due to both increased brilliance and coherence. We shall begin with the imaging technique offering the coarsest spatial resolution, graduating through imaging techniques of increased spatial resolution.

Before considering particular imaging techniques in detail, we briefly consider the phenomenon of diffraction. Diffraction generally limits the ultimate resolution of any linear imaging system. We can use Eq. (12) to model the diffraction of a plane wave by a single slit centred on the origin. This is done by constructing a thickness function, $t\left(\boldsymbol{x}_{\perp}\right)$, which is zero for $\left|x_{1}\right|<W / 2$ (i.e., let $\left.\boldsymbol{x}_{\perp}=\left(x_{1}, x_{2}\right)\right)$ and equal to some thickness, $T$, otherwise. If we then also select a large value of $\beta$ such that $\exp \left(-k_{0} \beta T\right) \approx 0$, Eq. (12) can be written as

$$
\Psi_{t}(\boldsymbol{r})=\frac{\exp \left(i k_{0} z\right)}{\sqrt{i z \lambda}} \int_{-W / 2}^{W / 2} \exp \left(i k_{0}\left(r_{1}-x_{1}\right)^{2} /(2 z)\right) \mathrm{d} x_{1}
$$




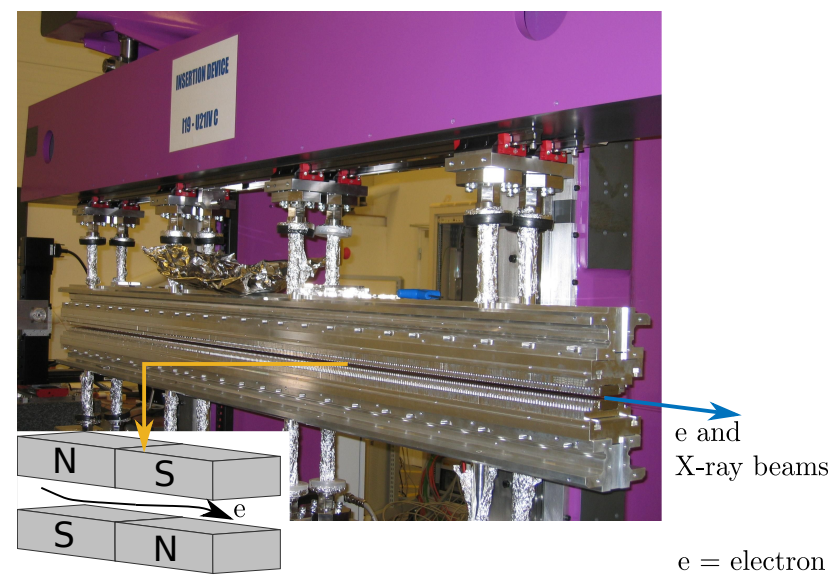

Figure 7: Photograph of an undulator used at the Diamond Light Source (Copyright Diamond Light Source) and a schematic diagram of the magnets making up the undulator and the path of the electrons through the undulator.

where, recall, $\boldsymbol{r}=\left(\boldsymbol{r}_{\perp}, z\right)=\left(r_{1}, r_{2}, z\right)$. This integral cannot, in general, be evaluated analytically, although some insight can be obtained by using the stationary phase approximation [28], due to the highly oscillatory nature of the integrand. We shall consider a model example to illustrate the key principles. Figure 8 shows the intensity of a plane wave, of wavelength $1.25 \AA$, which has been diffracted by a slit of width $W$, for five different values of $W$ ranging from $50 \mu \mathrm{m}$ down to $500 \mathrm{~nm}$. The nature of the intensity pattern at $z=0.5 \mathrm{~m}$ reveals much about the phenomenon of diffraction. For $W=50 \mu \mathrm{m}$, the intensity pattern at $z=0.5 \mathrm{~m}$ is seen to closely resemble the slit itself. Thus, imaging objects with a length scale matching the slit requires nothing more than an X-ray detector with suitably small pixels. The intensity pattern still resembles the slit itself for $W=15.8 \mu \mathrm{m}$, albeit with some perturbation. The situation is entirely different, however, for $W=500 \mathrm{~nm}$, where the field at $z=0.5 \mathrm{~m}$ does not resemble the slit at all. In fact, the field resembles the squared modulus of the Fourier transform of a rect function of width $W$. Thus, if $W$ were reduced further, the intensity pattern at $z=0.5 \mathrm{~m}$ would spread further in the lateral direction. Thus, measuring the width of such narrow slits would require sampling the diffraction pattern on increasingly wide sampling regions.

The concept of Fresnel number, denoted by $N_{F}$ [18] is a useful figure of merit for quantifying the regime in which a diffracting system operates. The Fresnel number has been calculated and displayed for each slit in Fig. 8 according to $N_{F}=W^{2} /(4 \lambda z)$. As can be seen, for large values of $N_{F}\left(N_{F} \gtrsim 1\right)$, the diffracted field resembles the slit itself, and geometrical optics remains valid. For $N_{F}$ much less than unity, the diffraction pattern becomes much wider than the slit and is found by Fourier transforming a rect function of width matching that of the slit. Intermediate values of $N_{F}$ result in a field which can only be described by evaluating Eq. (20).

We conclude this section with a word on coherence. As discussed previously, we assume 
perfect temporal coherence in this paper. So far in this example, we have also considered perfect spatial coherence since the illuminating wave was a plane wave, normally incident upon the slit. The concept of coherent modes discussed in Sec. (2.2) can be used to illustrate how partial coherence affects image formation. In particular, we have so far assumed that the illuminating field is represented by a single coherent mode, a plane wave at normal incidence. It would be possible, theoretically at least, to have a partially coherent field represented by multiple coherent modes, each of which could be a plane wave with a varying angle of incidence. Equation 19 then reveals that the intensity of the diffracted field will be the superposition of intensity patterns due to each plane wave. For small angles of incidence, as will usually be the case, the diffraction pattern simply shifts in accordance with the angle of incidence of the illuminating wavefield. Thus, if multiple plane wave coherent modes are present, the observed intensity will be a smeared-out version of that due to a single plane wave. Finally, we note that the coherent modes associated with real beamlines have a more complex structure than plane waves (see for example [29]), however this idealised treatment is sufficient to illustrate the impact of partial coherence on imaging.

\subsection{Macroscopic imaging}

\subsubsection{Propagation based imaging}

The first macroscopic imaging technique that we will consider is arguably the simplest. It was motivated by the observation of artefacts in images acquired in the early thirdgeneration synchrotron experiments [3]. This method, known as propagation based imaging (PBI), requires no dedicated hardware or image reconstruction algorithms beyond that of conventional X-ray imaging. The basic principle of PBI is illustrated in Fig. 9a), which shows schematically how a plane wave is perturbed by a non-absorbing wedge of refractive index $1-\delta$. The intensity of X-rays some distance down stream of the wedge is the result of the interference from three sources: a plane wave component not perturbed by the wedge, a plane component which is refracted by the wedge and a cylindrical wave originating from the tip of the wedge. Coherent addition of these three fields explains the nature of the fringes depicted in the plot of intensity in Fig. 9a).

We can adapt the ideas used to derive Eq. (20) to develop a simple illustrative description of PBI. Instead of the wedge in Fig. 9, consider a small block of width $W$ and depth $D$. The field in the $x y$-plane immediately after the block will be just the incident wave, everywhere except in the shadow of the block. In this shadow region, the field will be equal to the incident wave multiplied by the complex transparency of the block, $\exp \left(-i k_{0} \delta D\right) \approx 1-i k_{0} \delta D$. So the field immediately after the block is the superposition of the unperturbed incident plane wave (corresponding with the 1 ) and scattered field (corresponding with the $-i k_{0} \delta D$ ) emerging from the block. This scattered field is, thus, equivalent to the field diffracted by a slit of width $W$, as considered in Sec. (3), multiplied by $-i k_{0} \delta D$. Fringes due to interference between the incident and scattered fields are weak near the block because the incident and scattered 


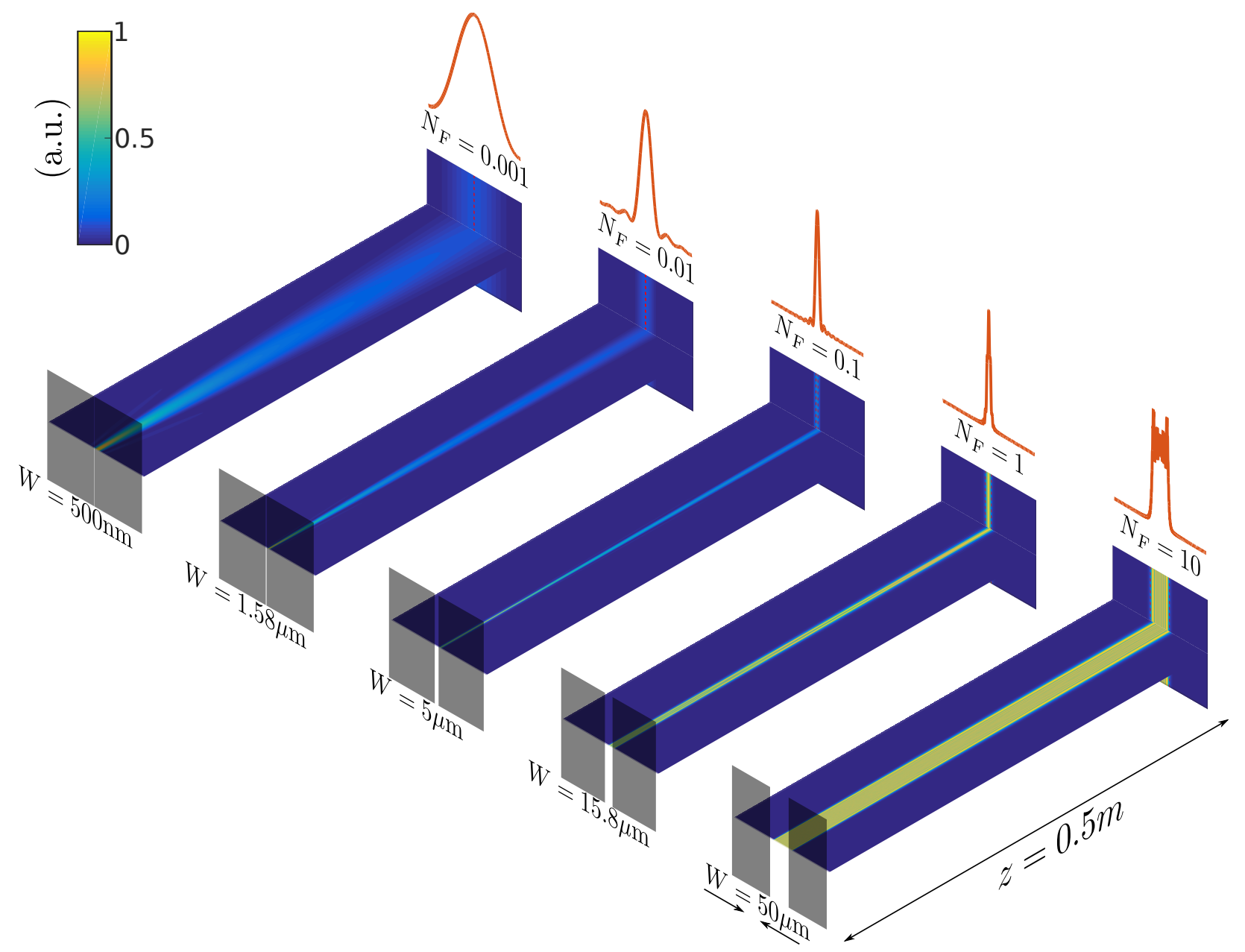

Figure 8: Illustration of diffraction of a plane wave by a single slit, for Fresnel numbers $\left(N_{F}\right)$ spanning four orders of magnitude. A photon energy of $9.93 \mathrm{keV}$, corresponding to a wavelength of $1.25 \AA$, was assumed. Field intensity is displayed and normalised by the maximum across all cases. Individually normalised line plots of intensity are also shown.

fields are initially out of phase due to the $-i$ term in the block's complex transparency function. If the block is narrow compared to the wavelength, the scattered field resembles that of a slit with a very small Fresnel number, such as the $N_{F}=0.001$ case in Fig. 8 . The field diffracted by the narrow slit is seen to diverge away from the projection of the slit with propagation distance from the slit. This divergence causes the diffracted field to have curved phase fronts (i.e., fronts of equal phase) which cross the plane phase fronts of the incident plane wave, leading to the generation of interference fringes. The principle of PBI is thus that propagation leads to stronger interference between scattered and directly incident fields.

For a more general scenario for a sample of thickness $t\left(\boldsymbol{x}_{\perp}\right)$ and refractive index $n=1-\delta+i \beta$, so long as $k_{0} \delta t\left(\boldsymbol{x}_{\perp}\right) \ll 1$ and $k_{0} \beta t\left(\boldsymbol{x}_{\perp}\right) \ll 1$, it can be shown that the intensity of X-rays 
downstream of the sample is well approximated by [6]

$$
I(\boldsymbol{r})=1-2 k_{0} \beta t\left(\boldsymbol{r}_{\perp}\right)-z \delta \nabla^{2} t\left(\boldsymbol{r}_{\perp}\right)
$$

which shows that the resulting image is the conventional absorption image enhanced with a signal proportional to the Laplacian of the sample's projected phase. Since we have assumed a homogeneous object, the object's projected phase is proportional to its thickness.

High spatial coherence is a prerequisite for performing PBI, and thus the reason why contrast due to $\delta$ was observed only after the advent of third-generation synchrotrons. Using the same argument as in the single slit diffraction example of Fig. 8, a source with limited spatial coherence will be represented not by a single coherent mode (i.e. a plane wave in the current case), but a superposition of coherent modes. In a highly idealised case, a source could be represented by plane wave coherent modes, in which case, the resulting intensity pattern would consist of the superposition of shifted intensity patterns each due to a single plane wave. The interference pattern in the perfectly coherent case possesses very fine fringes, due to the presence of the Laplacian in Eq. (21). Partially coherent illumination thus blurs, or in some cases completely annihilates, this interference pattern.

The first PBI images were published by Snigirev et al. [2] and soon afterwards by Cloetens et al. [3]. Since that time, the field has advanced significantly in multiple directions in, for example, clinical imaging [30], pre-clinical imaging [31] and entomology [32]. An image from the clinical mammography program based at the SYRMEP beamline of Elettra, the synchrotron radiation facility in Trieste, Italy, is shown in Fig. 10. The image is of an entire human breast imaged in-vivo [33-35]. One of the defining features of this image is the increased contrast of calcifications, the small bright dots scattered throughout the image. The calcifications have a high clinical significance and an imaging technique which is more sensitive to them is thus important. We conclude this section by noting that much work has also been devoted to performing quantitative imaging using PBI, the basis of which will be considered in Sec. (3.2.2).

\subsubsection{Analyser based imaging}

Highly coherent beamlines, which are also highly brilliant, allow the use of analyser crystals to perform phase contrast imaging. Analyser crystals are made from near-perfect crystals and use Bragg diffraction, due to the periodic arrangement of atoms within the crystal. This allows for plane waves to be selectively reflected within a narrow range of incident angles. Furthermore, within this narrow range, the crystal's reflectivity is highly sensitive to the angle of incidence, as is shown schematically in Fig. 9b). The approximately Gaussian reflectivity profile is often referred to as the "rocking curve". A monochromator crystal is placed before the sample to monochromatise the beam. An analyser crystal can then be used to sense sample induced refraction as shown in Fig. 9b). For example, suppose that the analyser crystal is oriented such that X-rays directly incident on the crystal experience 
a)

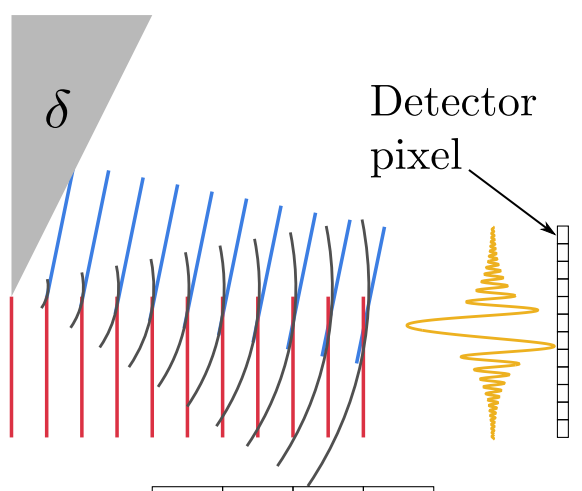

b)

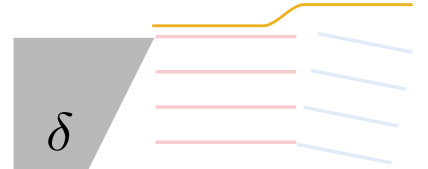

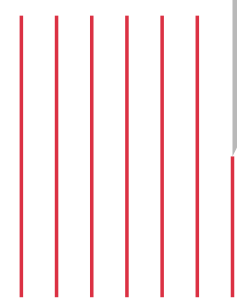

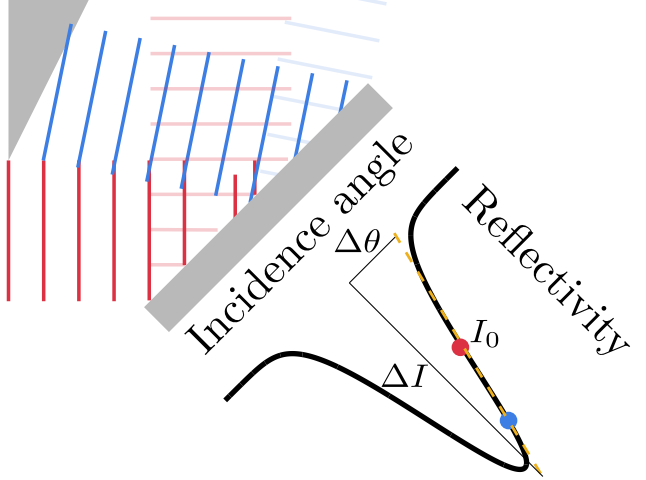

c)

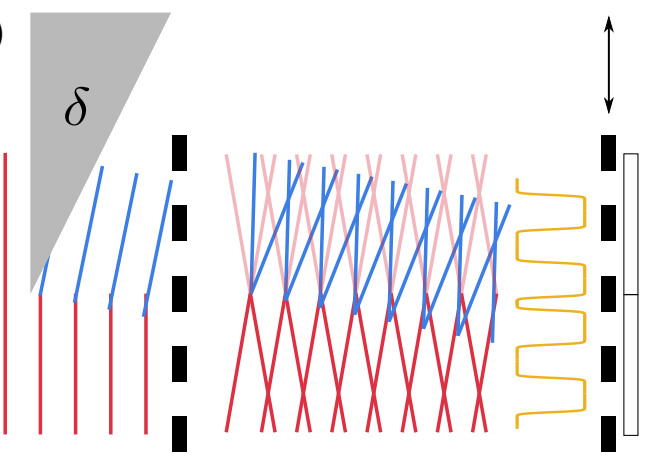

d)

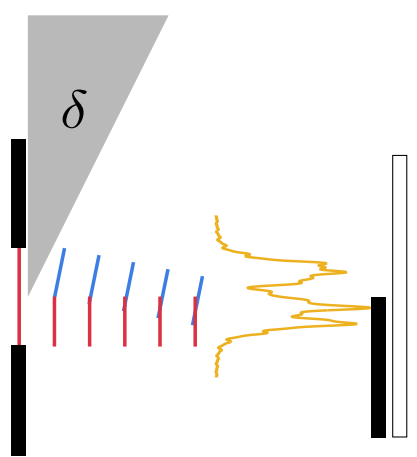

Figure 9: Schematic representations of the operating principles of propagation based imaging (PBI) (a), analyser based imaging (ABI) (b), grating interferometry (GI) (c) and edge illumination (EI) (d). In each case the signal rendered in yellow represents the irradiance of the field incident upon the detector.

a reflectivity of $50 \%$. If the rocking curve has an approximately linear region, as indicated by the dashed line in Fig. 9b), the amplitude of plane waves reflected by the crystal will be linearly proportional to the change in incident angle induced by a sample. Analyser based imaging (ABI) has been demonstrated to be very effective in imaging biological tissue [36]. Much effort has been devoted to performing quantitative imaging, where the known slope of the rocking curve $\Delta I / \Delta \theta$ (see Fig. 9b)) is exploited to separate absorption and refraction effects by acquiring two images, each on a different side of the rocking curve $[37,38]$. The high spatial coherence requirement for this method can be appreciated from the high sensitivity to incidence angle that the crystal reflectivity has. In particular, if the field incident upon the sample is not well described by a single monochromatic plane wave, the method's contrast will be significantly, if not entirely, compromised. It is also worth pointing out that this method requires highly monochromatic radiation, since the crystal rocking curve is very sensitive to wavelength. 


\subsubsection{Grating interferometry}

Grating interferometry (GI) makes use of the Talbot self-imaging phenomenon by which the image of a grating is replicated downstream of the grating, without the use of lenses. This phenomenon harnesses, rather than overcomes, the effects of diffraction by a grating. The Talbot phenomenon can be understood by using a mathematical model similar to that used to describe diffraction by a single slit in Eq. (20). This time, however, we consider diffraction by an infinite number of very narrow slits, equally spaced by a distance $L$. We can thus re-write Eq. (20) for this scenario as

$$
\begin{aligned}
\Psi_{t}(\boldsymbol{r}) & =\frac{\exp \left(i k_{0} z\right)}{\sqrt{i z \lambda}} \int_{-\infty}^{\infty}\left[\sum_{j=-\infty}^{\infty} \delta\left(x_{1}-j L\right)\right] \exp \left(i k_{0}\left(r_{1}-x_{1}\right)^{2} /(2 z)\right) \mathrm{d} x_{1} \\
& =\frac{\exp \left(i k_{0} z\right)}{\sqrt{i z \lambda}} \exp \left(i k_{0} r_{1}^{2} /(2 z)\right) \int_{-\infty}^{\infty}\left[\sum_{j=-\infty}^{\infty} \delta\left(x_{1}-j L\right)\right] \exp \left(i k_{0} x_{1}^{2} /(2 z)\right) \exp \left(-i k_{0} r_{1} x_{1} / z\right) \mathrm{d} x_{1} \\
& =\frac{\exp \left(i k_{0} z\right)}{L} \sum_{j=-\infty}^{\infty} \exp \left(-i \pi \lambda z j^{2} / L^{2}\right) \exp \left(i 2 \pi j r_{1} / L\right)
\end{aligned}
$$

where Fourier theory is used to go from Eq. (22) to Eq. (24). If we choose $z$ satisfying $z=2 n L^{2} / \lambda$, for some positive integer $n$, the quadratic phase term in Eq. (24) becomes equal to unity. We then use a further result from Fourier theory to write

$$
\Psi_{t}(\boldsymbol{r})=\frac{\exp \left(i k_{0} z\right)}{L} \sum_{j=-\infty}^{\infty} \delta\left(j-r_{1} / L\right)
$$

which shows that at Talbot distances (i.e. $z=2 n L^{2} / \lambda$ ), for this highly idealised problem, the structure of the grating is replicated by the structure of the field. The theory of Talbot self-image formation can be generalised for gratings employing transmitting regions with finite openings and also for phase gratings [39]. The mathematical basis of GI image formation can then be understood by inserting a wedge whose thickness, under the projection approximation (see Eq. (12)), is given by $t\left(\boldsymbol{x}_{\perp}\right)=\delta \alpha x_{1}+t_{0}$, where $t_{0}$ is set to ensure the thickness is positive in the region of interest, as must be the case physically. Insertion of an additional complex phase term, $\exp \left(-i k_{0} \delta \alpha x_{1}\right) \exp \left(-i k_{0} \delta t_{0}\right)$, into Eq. (22) is easily evaluated by defining $r_{1}^{\prime}=r_{1}+\delta \alpha z$ in Eq. (23), which causes Eq. (25) to become $\Psi_{t}(\boldsymbol{r})=\frac{\exp \left(i k_{0} z\right)}{L} \exp \left(-i k_{0} \delta t_{0}\right) \sum_{j=-\infty}^{\infty} \delta\left(j-\left(r_{1}+\delta \alpha z\right) / L\right)$. This shows that the self-image of the grating is simply shifted by an amount proportional to the phase gradient of the sample, $\delta \alpha$.

Typical grating pitches which have been used are between $4 \mu \mathrm{m}$ [40] and $8 \mu \mathrm{m}$ [41] which means that, in general, an additional grating must be employed as an analyser as shown in Fig. 9c). This second grating, usually termed the "analyser grating", has an absorption pattern which matches the Talbot pattern, and a period which is smaller than the detector pixel. It is then possible to extract both absorption and refraction images by sampling each pixel for several positions of the analyser grating [40]. 


\subsubsection{Edge illumination}

The final class of macroscopic phase contrast imaging methods is the so-called edge illumination (EI) technique shown schematically in Fig. 9d). This technique is conceptually similar to ABI due to the use of an absorbing edge in front of the detector to induce sensitivity to refraction, as is shown in Fig. 9d). This method is generally considered to be non-interferometric. This is illustrated by considering a representative example possessing apertures of width $20 \mu \mathrm{m}$ and a propagation distance of $0.55 \mathrm{~m}$ [42]. This setup yields a Fresnel number of $N_{F}=2.93$, at a photon energy of $20 \mathrm{keV}$, which Fig. 8 demonstrates to be in the domain where geometrical optics is applicable. This compares with grating interferometry which, if represented as employing apertures of width equal to half of the grating pitch, can be shown to have $N_{F} \leq 1 /(32 n)$ at the $n$th $(n>0)$ Talbot distance. This places GI in the domain where wave optics, as opposed to geometrical optics, must be used to describe the propagation of the field, as is also illustrated in Fig. 8.

A typical synchrotron realisation of an EI imaging system is shown in Fig. 11. The apertures in this system extended uniformly, within the beam, in the horizontal direction, so that the horizontal direction in Fig. 11 corresponds to the direction pointing out of the page in Fig. 9c). A strip detector was used with pixels arranged horizontally along the beam. Each acquisition of the detector thus generates the EI data corresponding to one row of an image. A complete image is generated by vertically scanning the sample through the beam.

Image formation for the EI method can be described by using the expression for diffraction by a single slit, Eq. (20), and inserting the same phase object used in the GI analysis after the single slit [43]. In a manner analogous to the GI case, the field diffracted by the aperture is shown to shift laterally at the detector by an amount $\delta \alpha z$. It is important to note, however, that the resolution of this method is not, in general, limited by the aperture width, since only photons in close proximity to the edge of the aperture partially blocking the pixel shown in Fig. 9d) contribute to image formation. In its simplest form, EI acquires images with mixed phase and absorption contrast, obtained by obstructing half of the pixel as is shown in Fig. 9d). An example of such an image, acquired by Diemoz et al. [44] of a flower petal and pollen grains, is shown in Fig. 12a). If, however, two images of the same sample are taken, one with each half of the pixel in Fig. 9d) obstructed, quantitative phase and absorption images can be obtained [43]. Figure 12b) shows such an example of the projected electron density corresponding with the mixed image in Fig. 12a). These images were acquired at beamline I13 (coherence branch) of the Diamond Light Source (Didcot, UK) at an X-ray energy of $9.7 \mathrm{keV}$. The pre-sample aperture had a width of $3 \mu \mathrm{m}$. Due to the unusually narrow pre-sample aperture, a high resolution pixelated detector was employed instead of a detector aperture. The sample was placed $5 \mathrm{~cm}$ from the pre-sample aperture and the detector was placed a further $30 \mathrm{~cm}$ downstream of the sample. 


\subsection{Microscopic imaging}

The macroscopic imaging techniques discussed in Sec. (3.1) all have a spatial resolution on the order of $10 \mu \mathrm{m}$. The spatial resolution of each method can be improved beyond standard configurations. However, as illustrated in Fig. 8, diffraction ultimately limits the spatial resolution that can be achieved. The diffraction limit is well known in optical microscopy and is proportional to $\lambda / \sin (\alpha)$, where $\alpha$ is the largest angle that a ray can make with the optical axis and still be accepted, or focused, by a lens. This is demonstrated in Fig. 13 where a zone plate is used in place of a refractive lens, as will be explained in Sec. (3.2.1). One might consider the macroscopic methods to have a very small value of $\alpha$, making $\lambda / \sin (\alpha)$ very large compared with $\lambda$. The remaining methods that we consider use either a lens, as in optical imaging, or computational techniques to increase the effective value of $\alpha$, and thus achieve improved spatial resolution.

\subsubsection{X-ray microscopy using lenses}

The translation of optical microscope design into the X-ray regime was not straight forward, due to both the lack of lenses and coherent X-ray sources. As discussed, coherent X-ray sources have developed significantly over recent decades. Compound refractive X-ray lenses have been demonstrated [46] with a focal length of the order of $1 \mathrm{~m}$. Such a focal length was achieved by combining 30 individual lenses having a focal length of the order of $50 \mathrm{~m}$. This extraordinarily long focal length is due to the $\delta$ values of most materials being less than $10^{-6}$ at X-ray wavelengths. Since then, focal lengths on the order of $1 \mathrm{~mm}$ have been demonstrated [47]. A disadvantage of refractive lenses is that they attenuate the the X-ray beam, thus reducing the number of photons available for imaging. Diffractive lenses, known as zone plates, were first demonstrated by Baez [48] for X-ray radiation and have proven to be more effective than refractive lenses at lower X-ray photon energies. The development of synchrotron radiation sources allowed for microscopes based on zone plates to be constructed $[49,50]$.

Frits Zernike developed a method of performing phase sensitive optical microscopy, for which he was awarded the Nobel Prize for physics in 1953 [51]. Translation of this technique into the X-ray regime is non-trivial given the need for both highly coherent X-ray sources and optical elements fabricated with micron precision. The principle of Zernike's method with Xray radiation is shown in Fig. 13. Zone plates, as depicted schematically in Fig. 13, are made up of concentric rings, alternating between transparent and opaque. The radial position of the $n$th boundary between rings is given by $\sqrt{n \lambda f}$ where $f$ is the focal length of the zone plate. This relationship can be found by considering Eq. (12) evaluated at the focus, i.e., $\boldsymbol{r}=(0,0, f)$. The ring boundaries are given by the values of $\boldsymbol{x}_{\perp}$ where the phase term in Eq. (12) undergoes a $\pi$ phase shift. This leads us to the relationship $k_{0}\left|\boldsymbol{x}_{\perp}\right|^{2} /(2 f)=n \pi$. This relationship thus ensures the constructive interference of X-rays at the focus of the zone plate. 
The operating principle of wide-field X-ray microscopes is identical to optical wide-field microscopes and is depicted in Fig. 13. In the absence of the element labeled "phase ring", the zone plate focuses X-rays diffracted by the sample onto a pixelated detector, where the image of the sample is formed. A condenser, which may be a zone plate, is used to illuminate the sample. When the phase ring in Fig. 13 is in place, the microscope becomes a phase contrast microscope. A variety of related implementations have been reported (see, for example [52-54]), and we report here a schematic representation. The zone plate in Fig. 13 can be thought of simply as a thin lens for the purposes of this explanation. The X-ray illumination incident upon the sample is delivered within a hollow cone. This is so that the non-diffracted illumination field is imaged onto the phase ring located at the back focal plane of the zone plate. In contrast to the non-diffracted illumination field, the X-rays diffracted by the sample fill the back focal-plane of the zone plate. This arrangement ensures that the non-diffracted field is perturbed by the phase ring, whereas the diffracted field is not. To understand the function of the phase ring, consider how a sphere, located at the origin, with refractive index $1-\delta$ and radius $R$, perturbs an incident field $U_{i}$. Under the projection approximation discussed in Sec. (2.1), particularly in relation to Eq. (12), we can say that the field immediately after the sphere is given by $U_{s}=U_{i} \exp \left(-i k_{0} \delta t\left(\boldsymbol{x}_{\perp}\right)\right) \approx U_{i}\left(1-i k_{0} \delta t\left(\boldsymbol{x}_{\perp}\right)\right)$ where $t\left(\boldsymbol{x}_{\perp}\right)=2 \sqrt{R^{2}-\left|\boldsymbol{x}_{\perp}\right|^{2}}$ for $\left|\boldsymbol{x}_{\perp}\right| \leq R$. The field $U_{s}$ is thus seen to be composed of two components, the non-diffracted field, $U_{i}$, and the diffracted field $-U_{i} i k_{0} \delta t\left(\boldsymbol{x}_{\perp}\right)$. This analysis demonstrates that the non-diffracted and diffracted fields are $\pi / 2$ out of phase with one another, which is why phase contrast is, in general, weak in attenuation based imaging systems. Zernike made his great advance by realising that this contrast can be enhanced by phase shifting the non-diffracted field so that it is in-phase with the diffracted field. This phase shifting is achieved by the phase ring which has a thickness, $D_{p r}$ (see Fig. 13) and $\delta=\delta_{p r}$ such that $k_{0} D_{p r} \delta_{p r}=\pi / 2$. This significantly enhances phase contrast due to the sample's $\delta$ distribution at the detector. In practice, the sample is rotated about an axis normal to the optical axis in order to perform three-dimensional imaging using computed tomography (CT).

Figure 14 contains images of a graphite electrode as found in a lithium-ion battery as reported in [55]. The images were acquired using a Zernike phase contrast X-ray microscope with a sufficiently coherent Rigaku Micromax-7 (Rigaku Corporation, Tokyo, Japan) laboratory source, with a $\mathrm{Cr}$ anode emitting photons at $5.4 \mathrm{keV}$, rather than a synchrotron. The X-ray microscope was a ZEISS Xradia 810 Ultra (Carl Zeiss X-ray Microscopy Inc., Pleasanton, CA). 3021 projection images of the electrode were taken, each for a different sample rotation angle covering $360^{\circ}$ of rotation, allowing a $3 \mathrm{D}$ volume to be reconstructed. Imaging was performed both in phase contrast and absorption contrast modes, and a single slice example of each of these is displayed in Fig. 14a) and b), respectively. Three-dimensional renderings are also shown in Fig. 14c) and d), which show the individual particles of graphite. The resolution of this X-ray microscope is quoted as $150 \mathrm{~nm}$ for this study, and can be as fine as $50 \mathrm{~nm}$ [55]. An obvious difference between these images and those of the EI method in Fig. 12 is that the scale bar is an order of magnitude larger for EI. One disadvantage of the $\mathrm{X}$-ray microscope is its limited field of view, typically less than $60 \mu \mathrm{m}$, which is due to the 
difficulty of fabricating large zone plates and detectors with a correspondingly large number of pixels.

We conclude this section by noting that, just as in optical microscopy, scanning microscopes have been developed. Scanning X-ray microscopes work by illuminating the sample with a very narrow beam of X-rays, then using a single pixel detector to detect the total amount of X-rays transmitted by the sample. Such a system [50] can be constructed by using a zone plate, as depicted in Fig. 13, to focus light onto a small circular aperture. The sample is positioned immediately after the aperture, and a wide area detector placed to collect the majority of X-rays transmitted by the sample. This is conceptually similar to the EI system depicted in Fig. 9d), except that a single large area detector is placed in close proximity to the sample. An image is built up by sampling the detector signal for different positions of the sample relative to the aperture.

\subsubsection{Lensless coherent diffractive imaging}

We discussed in Sec. (3.2.1) how lenses can be used to perform X-ray microscopy. One of the challenges of this approach is the need to make high precision optical elements and maintain the alignment of such elements. An alternative to this approach is to use computational techniques to calculate the sample structure causing a particular diffraction pattern to be observed. This type of imaging is usually called coherent diffractive imaging (CDI). Consider, for example, the arrangement shown in Fig. 15a), where a plane wave is incident upon a sample, and a detector records the resulting diffraction pattern. Other system geometries are possible. See [7] for a good overview of the different approaches to CDI. The geometry depicted in Fig. 15a) is both simple and representative of the other geometries. The computational problem of lensless imaging is, in terms of Eq. (12), to determine $\delta, \beta$ and $t\left(\boldsymbol{x}_{\perp}\right)$ which causes a particular form of $\left|\Psi_{t}(\boldsymbol{r})\right|^{2}$ to be measured. If the distance between the sample and the detector, $z$, is large compared with $k_{0} D^{2} /(2 z)$, where $D$ is half the detector height as shown in Fig. 15a), the relationship between $\Psi_{t}(\boldsymbol{r})$ and $f\left(\boldsymbol{x}_{\perp}\right)=\exp \left(k_{0}(-i \delta-\beta) t\left(\boldsymbol{x}_{\perp}\right)\right)$ becomes a Fourier transform relationship which, ignoring a constant complex multiplier, may be written as

$$
\Psi_{t}(\boldsymbol{r})=\int_{-\infty}^{\infty} f\left(\boldsymbol{x}_{\perp}\right) \exp \left(-i k_{0} \frac{\boldsymbol{r}_{\perp} \cdot \boldsymbol{x}_{\perp}}{z}\right) \mathrm{d}^{2} \boldsymbol{x}_{\perp}
$$

The so-called phase problem can then be stated in terms of determining $f\left(\boldsymbol{x}_{\perp}\right)$ given measurements of $\left|\Psi_{t}(\boldsymbol{r})\right|^{2}$. The solution to this problem has been the subject of a great deal of research since the seminal work of Gerchberg and Saxton [56]. A good review of this work is given by Fienup [57]. Gerchberg and Saxton [56] demonstrated an algorithm for solving the phase problem assuming that $\left|\Psi_{t}(\boldsymbol{r})\right|^{2}$ is known for both large and small values of $z$, i.e., that the diffraction and direct images of the sample are known. The algorithm works by iterating through subsequent estimates for $f\left(\boldsymbol{x}_{\perp}\right)$ until the error between the calculated and observed values of $\left|\Psi_{t}(\boldsymbol{r})\right|^{2}$ goes below a particular threshold. This approach is not 
appropriate for application in CDI since the sample is usually so small that the direct image is unable to be obtained. The Gerchberg Saxton algorithm was modified by Fienup to work when the support of the sample (i.e., where its thickness, $t\left(\boldsymbol{x}_{\perp}\right)$, is non-zero) is finite and known [58]. Significant work was done by Bates and co-workers in establishing the conditions under which the phase problem admits a unique solution [59-61], with the conclusion being that under most practical situations, a unique solution exists so long as the sample has finite support. Another important theoretical result is that the intensity pattern, $\left|\Psi_{t}(\boldsymbol{r})\right|^{2}$, is required to be sampled at twice its Nyquist frequency [62].

CDI is yet to realise its immense potential as a high resolution coherent $\mathrm{X}$-ray imaging technique, particularly in biology. This is most likely due to non-ideal experimental complications. One issue is that a beam stop must be placed in front of the detector to block the directly incident beam. This stops the detector from being saturated by the incident beam and also prevents it being damaged by radiation. This in turn creates a problem for the algorithm used to solve the phase problem as a central region of the diffraction pattern is not able to be measured. A more significant challenge, which has impeded the demonstration of compelling experimental results, is the imperfect coherence of third-generation synchrotron beamlines. The effect of this can be understood by recalling the coherent mode decomposition discussed in Sec. (2.2). We have until now, in this section, considered a single plane wave incident upon the sample, which admits the Fourier relationship expressed in Eq. (26). Perfect coherence means that our X-ray field is described by a single mode, $\phi_{1}$, in Eq. (18), which we have taken to be a plane wave. In the general case of partial coherence we must, however, write

$$
\left|\Psi_{t}(\boldsymbol{r})\right|^{2}=\sum_{n} \alpha_{n}\left|\Psi_{t, n}(\boldsymbol{r})\right|^{2}
$$

where the index, $n$, refers to the coherent mode number. This entirely changes the nature of the phase problem since the pure Fourier relationship is broken which turns out to be a fundamental problem [6]. Despite these challenges, there have been a number of experimental demonstrations since the first reported by Miao et al. [63]. Further examples include imaging of biomolecules [64] (compare this with the technique presented in Sec. (3.3)), nanostructures [65] and three-dimensional CDI tomography [65].

Just as in lens-based X-ray microscopy discussed in Sec. (3.2.1), scanning versions of CDI have emerged as shown schematically in Fig. 15b). Perhaps the first step, within the domain of X-ray imaging, towards this approach was taken by Abbey and co-workers [66], who developed so-called keyhole CDI, which allowed extended objects to be imaged using an illumination beam of finite size. This was followed closely by the demonstration of a technique known as ptychography [67], the translation of a technique developed for electron microscopy $[68,69]$ into the X-ray regime. Ptychography works by scanning a focused probe beam over the sample and recording the diffraction pattern for each location of the probe beam. The diffracted field can be found by modifying Eq. (26) to include the form of the 
probe beam, $\Psi_{i}\left(\boldsymbol{x}_{\perp}\right)$ as

$$
\Psi_{t}\left(\boldsymbol{r}_{j} ; \boldsymbol{r}\right)=\int_{-\infty}^{\infty} \Psi_{i}\left(\boldsymbol{x}_{\perp}-\boldsymbol{r}_{j_{\perp}}\right) f\left(\boldsymbol{x}_{\perp}\right) \exp \left(-i k_{0} \frac{\boldsymbol{r}_{\perp} \cdot \boldsymbol{x}_{\perp}}{z}\right) \mathrm{d}^{2} \boldsymbol{x}_{\perp},
$$

where $\left|\Psi_{t}\left(\boldsymbol{r}_{j} ; \boldsymbol{r}\right)\right|^{2}$ is acquired for a range of values of $\boldsymbol{r}_{j}$ covering the entire sample. Performing two-dimensional imaging will thus result in a four-dimensional data set. A key property of ptychography is that adjacent values of $\boldsymbol{r}_{j}$ are chosen to ensure a sufficient overlap between the two probe beam positions. The resulting redundancy in the data is important in the iterative reconstruction technique. A further important property is that ptychography allows for the probe beam to be extracted as part of the reconstruction [67]. This allows for non-idealities of the X-ray beam and focusing optics to be accounted for without degrading the resulting image. Another important property of ptychography is that a beam stop is not required, as in conventional CDI, since the illumination beam diverges after the sample, thus reducing in intensity before being incident on the detector.

Figure 16 shows images illustrating ptychographic imaging of a porous hydroxyapatite sphere [70]. The data for these images was acquired at the Swiss Light Source (cSAXS beamline) at a photon energy of $6.2 \mathrm{keV}$. The probe profile, reconstructed along with the object transmission function, is shown in Fig. 16c), and is approximately $2 \mu \mathrm{m}$ in width, which is clearly smaller than the sphere's diameter. Thus, 387 different diffraction patterns were acquired, for different probe positions relative to the sample. The size of each pixel in the transmission function images is $65.3 \mathrm{~nm}$. The reconstruction algorithm used to obtain the results in Fig. 16 employed maximum-likelihood principles to mitigate stochastic noise sources due to the random arrival of photons. This source of noise turns out to fundamentally limit the spatial resolution which may be obtained by microscopic imaging techniques. This is because increasing the number of photons diffracted by the sample generally requires increasing the radiation dose delivered the sample. This increased dose can damage the sample before sufficient diffracted photons have been detected. This phenomenon has been demonstrated to fundamentally limit the spatial resolution achievable in X-ray microscopy [71]. As is discussed in the next section, however, the advent of free electron lasers has provided a way to overcome this problem by bombarding the sample with photons in such a short time that the required number of diffracted photons can be detected before radiation damage has occurred $[72]$.

\subsection{Molecular scale imaging}

The final imaging technique that we consider represents the state of the art in coherent X-ray imaging in terms of resolution. It makes use of results from crystallography, a substantial field of research in its own right. We do not delve deeply into crystallography, however there are a number of good introductory texts on the subject such as those by Ashcroft and Mermin [73] and Hammond [74]. Crystallography has been integral in building our knowledge of the atomic structure of an extremely wide range of compounds. As discussed 
in Sec. (2.1), if $\rho(\boldsymbol{x})$ in Eq. (8) is a function periodic in three-dimensions, the diffraction pattern, i.e., the scattered field observed a long way from the crystal, is made up of sharp peaks known as Bragg peaks. The Bragg peaks are strong compared with the diffraction pattern of, for example, a single molecule, due to the constructive interference between the diffraction patterns of periodically repeated molecules. A crystal structure can be determined by solving the so-called phase problem of determining $\rho(\boldsymbol{x})$ from the location and intensity of the Bragg peaks, the solution of which is now very well understood.

One of the challenges of crystallography is growing a crystal of sufficient size, as the intensity of the Bragg peaks scales with the number of times each molecule is repeated within the crystal. If a crystal of sufficient size is synthesised, it may contain defects whereby the repeated molecules are imperfectly arranged within the crystal. We illustrate the principles of crystallography using a much simpler one-dimensional, infinitely-thin, slit diffraction geometry as discussed in Sec. (3.1.3). We first consider an arrangement of $N_{s}=10$ infinitely thin slits arranged randomly as in the boxed region of Fig. 17b). The term $\sum_{j=-\infty}^{\infty} \delta\left(x_{1}-j L\right)$ in Eq. (22) would be replaced by $\sum_{j=1}^{N_{s}} \delta\left(x_{1}-x_{j}\right)$, where $x_{j}$ is the randomly chosen position of the $j$ th slit. The so-called continuous diffraction pattern due to these $N_{s}$ slits is plotted in blue in Fig. 17a) and is repeated for reference in Figs. 17c) and e). Next, we consider a periodic arrangement of $N_{r}=51$ asymmetric units of the $N_{s}$ slits as also shown in Fig. 17b). The diffraction pattern for this periodic arrangement is plotted in red in Fig. 17a), noting that the red plot has an absolute value of $N_{r}$ times that of the blue continuous diffraction pattern. In this ideal case, the peaks in the periodic diffraction pattern, which are analogous to Bragg peaks, sample the continuous diffraction pattern with a magnitude scaled by the number of repeated asymmetric units $N_{r}$.

In more realistic cases, the asymmetric unit in a crystal may not be arranged with perfect periodicity. Continuing with the single slit analogy, we consider how the random displacement of each unit of $N_{s}$ slits affects the diffraction pattern. The diffraction pattern which arises when each of the $N_{r}$ units of slits experiences a random shift is plotted in red in Fig. 17c). The peaks in the diffraction pattern rapidly decay away from the origin giving way to highly oscillatory fringes. When considering imperfect crystals, this phenomenon limits the resolution to which the crystal structure is able to be solved since the maximum value of $r_{1}$, at which a peak is observable, determines the value of $\alpha$ discussed in Sec. (3.2) in the context of the diffraction limit.

A step towards recovering additional information from the diffraction patterns of small, imperfect, crystals was made recently by Kirian et. al [75]. They noted that if the diffraction patterns of many imperfect crystals are correctly averaged, the diffraction pattern of the molecule of interest can be recovered. Turning again to the slit analogy, instead of considering a single periodic extension of $N_{r}$ imperfectly repeated asymmetric units of slits, we consider an ensemble average of $10^{4}$ different random displacements of the repeated asymmetric units of slits. This averaged diffraction pattern is plotted in red in Fig. 17e), which shows that the peaks from the perfect periodic extension of the slits are present near the origin and a scaled version of the continuous diffraction pattern is present at larger values of $r_{1}$. We have 
presented an illustration of the principle employed by Ayyer et. al [4] to recover both the Bragg and continuous diffraction patterns from imperfect nanocrystals. They have provided a formal mathematical derivation of this principle for such crystals.

Having considered the principle of the method presented by Ayyer et. al [4], we now consider some details of the experiment. As has already been explained, the method uses two sources of information: the Bragg peaks associated with crystallography and the so-called continuous diffraction pattern associated with the single, asymmetric, molecule of interest. A large number of crystals were imaged, resulting in 61,946 diffraction patterns. The crystals flow in a jet through the X-ray beam and so the orientation is not known at the time of acquisition. The presence of some Bragg peaks in the diffraction patterns is, however, a significant advantage as existing databases of molecular structure can be used to infer the orientation of each crystal from its diffraction pattern. This information also allows the so-called continuous diffraction pattern corresponding to the single molecule to be correctly assigned. The result of this procedure of averaging correctly assigned diffraction patterns is a diffraction pattern with Bragg peaks at low scattering angles and a continuous diffraction pattern at higher scattering angles, in analogy with the red plot in Fig. 17e).

The phase problem, i.e., recovery of the molecule's electron density from the magnitude of the collated diffraction patterns, is solved in two stages. The Bragg diffraction data was initially used to determine the electron density to a resolution of $4.5 \AA$ using techniques established in crystallography. A method previously used in CDI (see Sec. (3.2.2)) was then used to refine this initial electron density calculation to a resolution of $2.2 \AA$, using the continuous diffraction data. This is possible because the continuous diffraction pattern extends further from the origin, increasing the effective value of $\alpha$ discussed in Sec. (3.2), thus refining the diffraction limited resolution of the imaging system.

The development of this imaging technique required the use of highly brilliant free electron lasers. It is very difficult to measure the diffraction pattern of small crystals because they diffract poorly. For a given crystal, the number of diffracted photons is proportional to the number of incident photons. So obtaining a satisfactory signal to noise ratio in the diffraction pattern requires a threshold of incident photons to be exceeded. Before the advent of Xray lasers, inferior source brilliance meant that by the time enough photons were incident on the crystal, the crystal had been severely damaged by radiation. The advent of free electron lasers, such as the linac coherent light source [76], meant that very short pulses (40fs duration as used by Ayyer et. al [4]) allow the required number of photons to interact with the crystals before radiation damage has occurred [72].

We conclude this section with an image, produced by Ayyer et. al [4], of photosystem II, a membrane protein complex responsible for the light activated splitting of hydrogen and oxygen atoms from water molecules [77]. Just a part of the electron density maps of the membrane protein complex is shown in Fig. 18 calculated using only Bragg information a) and using both the Bragg and continuous diffraction data b). The mesh in each image represents the contoured electron density map. Note that the mesh in b) is finer than in a). 
The importance of this is that the model building software used to determine the molecular structure is more accurate if the electron density map is more accurate. This represents the most accurate model available of this important membrane protein complex.

\section{Conclusions and future outlook}

Coherent X-ray imaging is anticipated to have a bright and exciting future. Whilst the most recent breakthroughs in molecular scale imaging are already very stimulating, the commissioning of more free electron lasers and the upgrade of synchrotron sources around the world will enable a plethora of novel research. New insights will be obtained by leveraging the spatial resolution, mixed contrast mechanisms and imaging speed made possible by coherent X-ray sources.

\section{A Appendix: derivation of equations describing X-ray interactions with matter on a macroscopic scale.}

We begin with Eq. (11) and define $\boldsymbol{x}_{\perp}$ and $\boldsymbol{r}_{\perp}$ such that $\boldsymbol{x}=\left(\boldsymbol{x}_{\perp}, \zeta\right)$ and $\boldsymbol{r}=\left(\boldsymbol{r}_{\perp}, z\right)$. Assuming that $z \gg \zeta$ allows us to write

$$
\begin{aligned}
\Psi_{s}(\boldsymbol{r}) & =-\left(f_{1}-i f_{2}\right) r_{0} \int_{-\infty}^{\infty} \int_{0}^{t} n_{a} \frac{\exp \left(i k_{0}|\boldsymbol{r}-\boldsymbol{x}|\right)}{|\boldsymbol{r}-\boldsymbol{x}|} \exp \left(i k_{0} \zeta\right) \mathrm{d}^{2} \boldsymbol{x}_{\perp} \mathrm{d} \zeta \\
& \approx-\left(f_{1}-i f_{2}\right) r_{0} n_{a} t \frac{\exp \left(i k_{0} z\right)}{z} \int_{-\infty}^{\infty} \exp \left(i k_{0} \frac{\left|\boldsymbol{r}_{\perp}-\boldsymbol{x}_{\perp}\right|^{2}}{2 z}\right) \mathrm{d}^{2} \boldsymbol{x}_{\perp} \\
& =-i \lambda\left(f_{1}-i f_{2}\right) r_{0} n_{a} t \exp \left(i k_{0} z\right)
\end{aligned}
$$

where we have used the results $\int_{-\infty}^{\infty} \exp \left(i k_{0}\left|\boldsymbol{x}_{\perp}\right|^{2} /(2 z)\right) \mathrm{d}^{2} \boldsymbol{x}_{\perp}=i \lambda z$. and $|\boldsymbol{r}-\boldsymbol{x}| \approx(z-$ $\zeta)+\left|\boldsymbol{r}_{\perp}-\boldsymbol{x}_{\perp}\right|^{2} /(2(z-\zeta))$. If we now add the scattered field expressed in Eq. (31) to the unscattered field, since we assume that $t$ is small, the total field may be obtained as

$$
\begin{aligned}
\Psi_{t}(\boldsymbol{r}) & =\exp \left(i k_{0} z\right)\left(1-i \lambda r_{0}\left(f_{1}-i f_{2}\right) n_{a} t\right) \\
& \approx \exp \left(i k_{0}\left(z-\frac{\lambda^{2} r_{0}}{2 \pi}\left(f_{1}-i f_{2}\right) n_{a} t\right)\right)
\end{aligned}
$$

If, alternatively, we employ a formalism based on the usual definition of the refractive index at X-ray energies, $n=1-\delta+i \beta$, the wave transmitted through such a slab of material would be given by

$$
\Psi_{t}(\boldsymbol{r})=\exp \left(i k_{0}(z-(\delta-i \beta) t)\right)
$$


Comparing Eqs. (33) and (32) allows us to conclude that

$$
\delta=\frac{\lambda^{2} n_{a} f_{1} r_{0}}{2 \pi} \quad \beta=\frac{\lambda^{2} n_{a} f_{2} r_{0}}{2 \pi} .
$$

Far from resonant frequencies, $f_{1}$ is well approximated simply by the average number of electrons per atom in a material [17], allowing us to write

$$
\delta=\frac{\lambda^{2} \rho r_{0}}{2 \pi}
$$

where $\rho$ is the material's electron density. This link between $\delta$ and electron density is important in the field of X-ray phase contrast imaging.

We are now able to derive some equations which are important to coherent X-ray imaging on a macroscopic scale. If we take the definitions of $\delta$ and $\beta$ in Eq. (34) and substitute them into Eq. (29), whilst allowing the sample thickness to vary with $\boldsymbol{x}_{\perp}$, instead of being a slab, we can write

$$
\begin{aligned}
\Psi_{s}(\boldsymbol{r}) & =\frac{\exp \left(i k_{0} z\right)}{i \lambda z} k_{0}(-i \delta-\beta) \int_{-\infty}^{\infty} \int_{0}^{t\left(\boldsymbol{x}_{\perp}\right)} \exp \left(i k_{0} \frac{\left|\boldsymbol{r}_{\perp}-\boldsymbol{x}_{\perp}\right|^{2}}{2 z}\right) \mathrm{d}^{2} \boldsymbol{x}_{\perp} \mathrm{d} \zeta \\
& =\frac{\exp \left(i k_{0} z\right)}{i \lambda z} k_{0}(-i \delta-\beta) \int_{-\infty}^{\infty} t\left(\boldsymbol{x}_{\perp}\right) \exp \left(i k_{0} \frac{\left|\boldsymbol{r}_{\perp}-\boldsymbol{x}_{\perp}\right|^{2}}{2 z}\right) \mathrm{d}^{2} \boldsymbol{x}_{\perp} \\
& \approx \frac{\exp \left(i k_{0} z\right)}{i \lambda z} \int_{-\infty}^{\infty}\left[\exp \left(k_{0}(-i \delta-\beta) t\left(\boldsymbol{x}_{\perp}\right)\right)-1\right] \exp \left(i k_{0} \frac{\left|\boldsymbol{r}_{\perp}-\boldsymbol{x}_{\perp}\right|^{2}}{2 z}\right) \mathrm{d}^{2} \boldsymbol{x}_{\perp} \\
& =-\exp \left(i k_{0} z\right)+\frac{\exp \left(i k_{0} z\right)}{i \lambda z} \int_{-\infty}^{\infty} \exp \left(k_{0}(-i \delta-\beta) t\left(\boldsymbol{x}_{\perp}\right)\right) \exp \left(i k_{0} \frac{\left|\boldsymbol{r}_{\perp}-\boldsymbol{x}_{\perp}\right|^{2}}{2 z}\right) \mathrm{d}^{2} \boldsymbol{x}_{\perp}
\end{aligned}
$$

\section{Acknowledgments}

PM is supported by a Royal Society University Research Fellowship. PM would like to acknowledge insightful discussions with members of the Advanced X-ray Imaging Group (University College London), Dr Chris Hall and Dr Andrew Stevenson (The Australian Synchrotron) and Dr Joe Ferrara (Rigaku Corporation). 


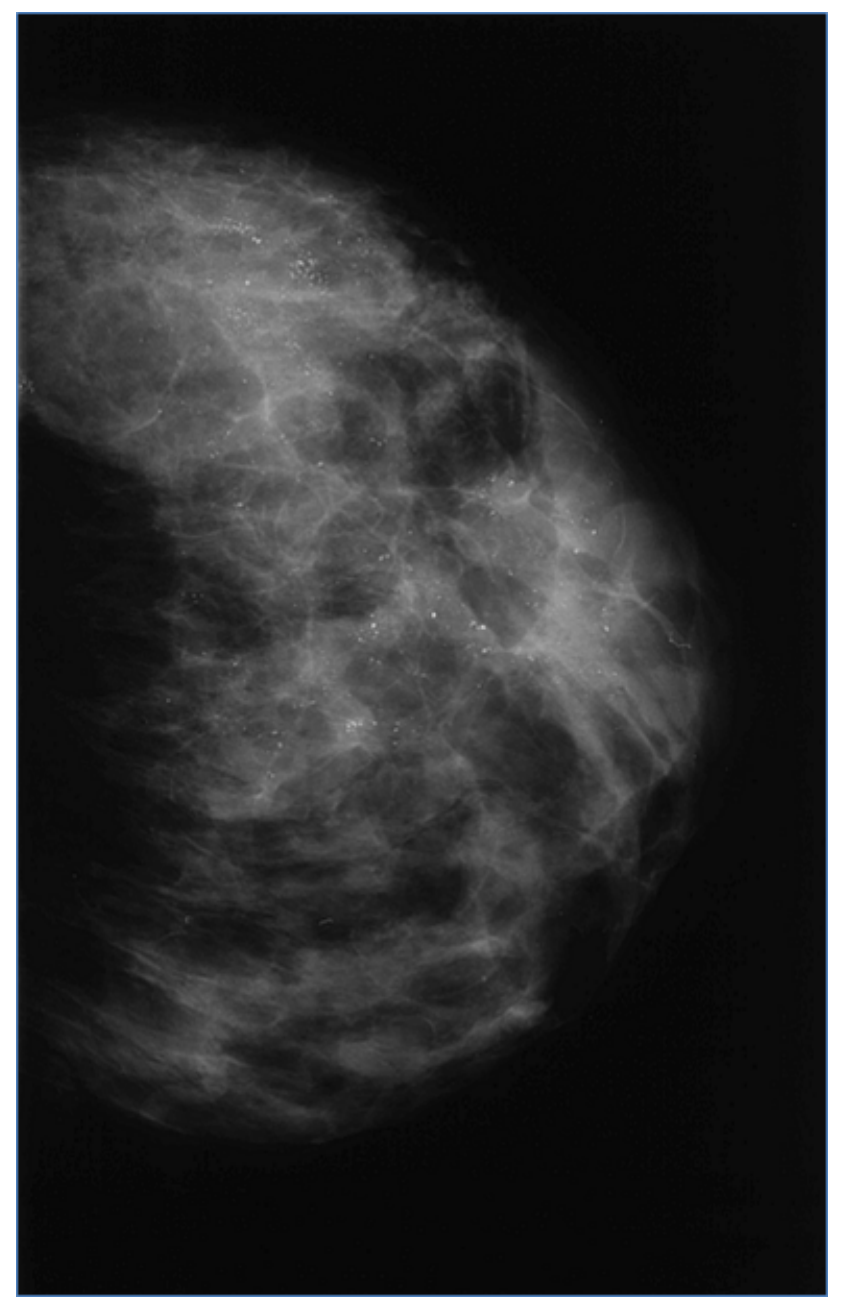

Figure 10: PBI image of a human breast acquired in-vivo, showing calcifications as bright dots throughout the image with much greater contrast than in conventional mammography. Printed with the Permission of R. Longo and acquired during the clinical program described in $[33-35]$. 


\section{Pre-sample}

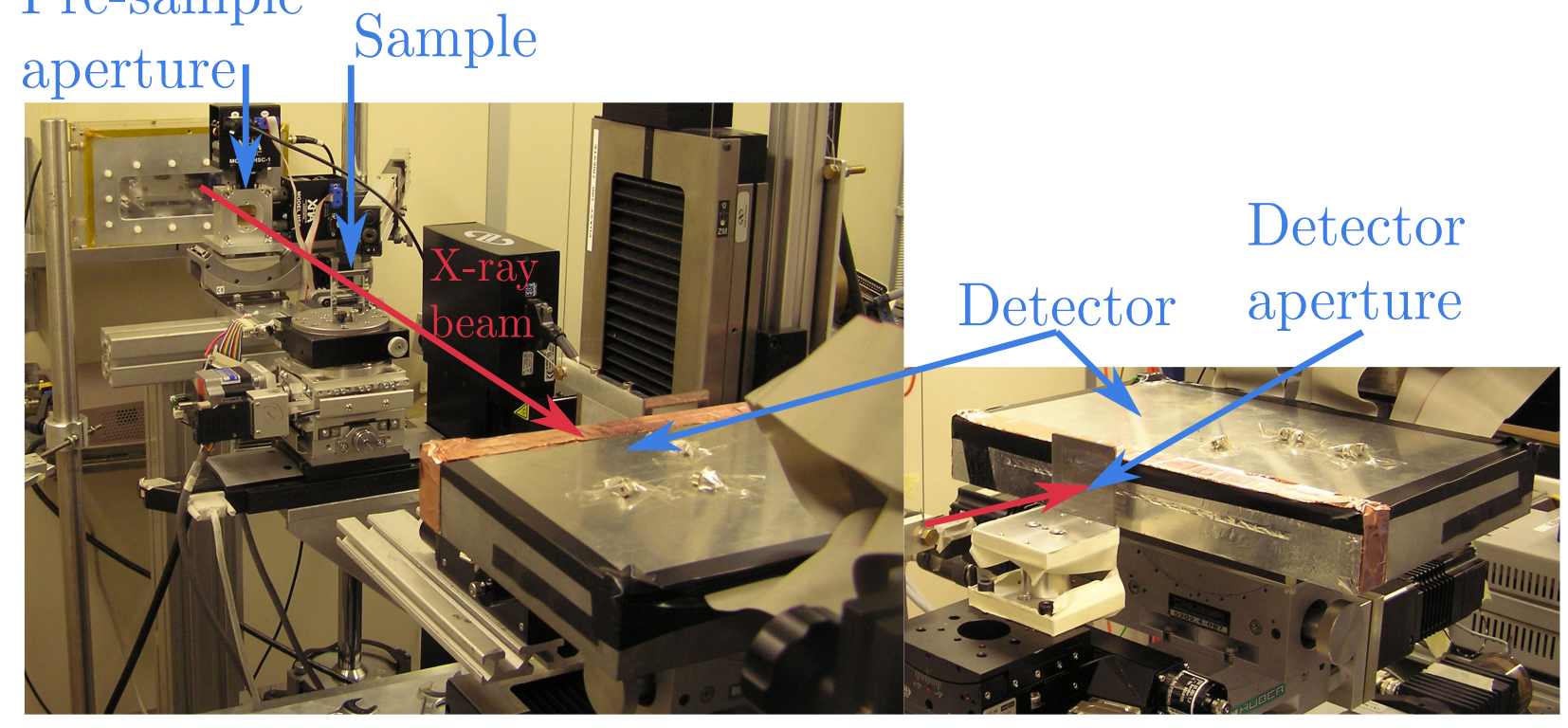

Figure 11: A photo of a typical edge illumination (EI) setup [45] like that depicted in Fig. $9 \mathrm{c}$ ). The image on the right shows the detector from a different angle, allowing the detector aperture to be seen. The system is sensitive to sample phase variations in the vertical direction. The sample had to be scanned in the vertical direction to obtain an image. The horiztonal direction in this image corresponds to the direction pointing out of the page in Fig. 9c). 


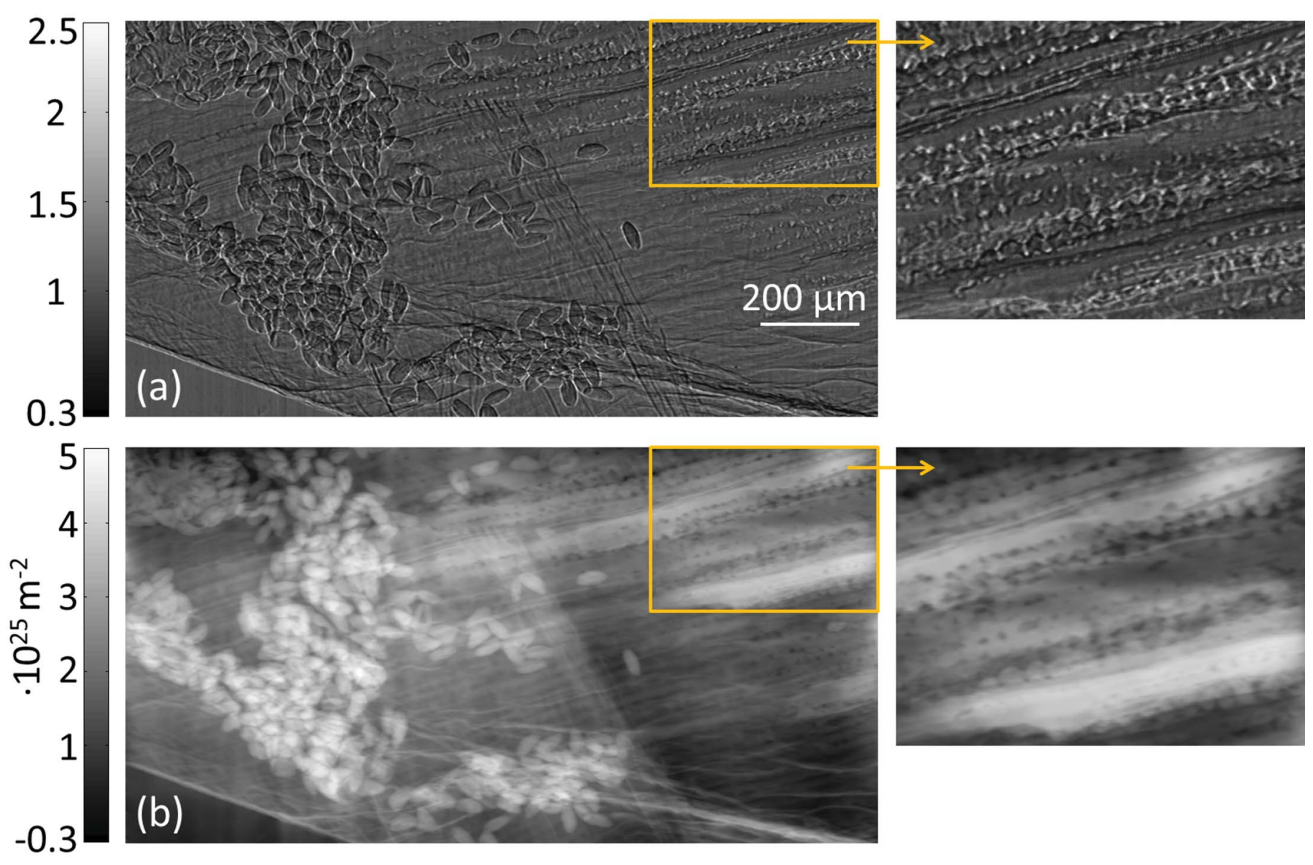

Figure 12: EI images of a flower petal and pollen grains: (a) mixed phase and absorption image, normalised by the detected intensity in the absence of a sample, (b) retrieved map of the projected electron density. Cells lining the petal are visible in the magnified regions. Reproduced with permission of the P. Diemoz and the International Union of Crystallography, derived from [44].

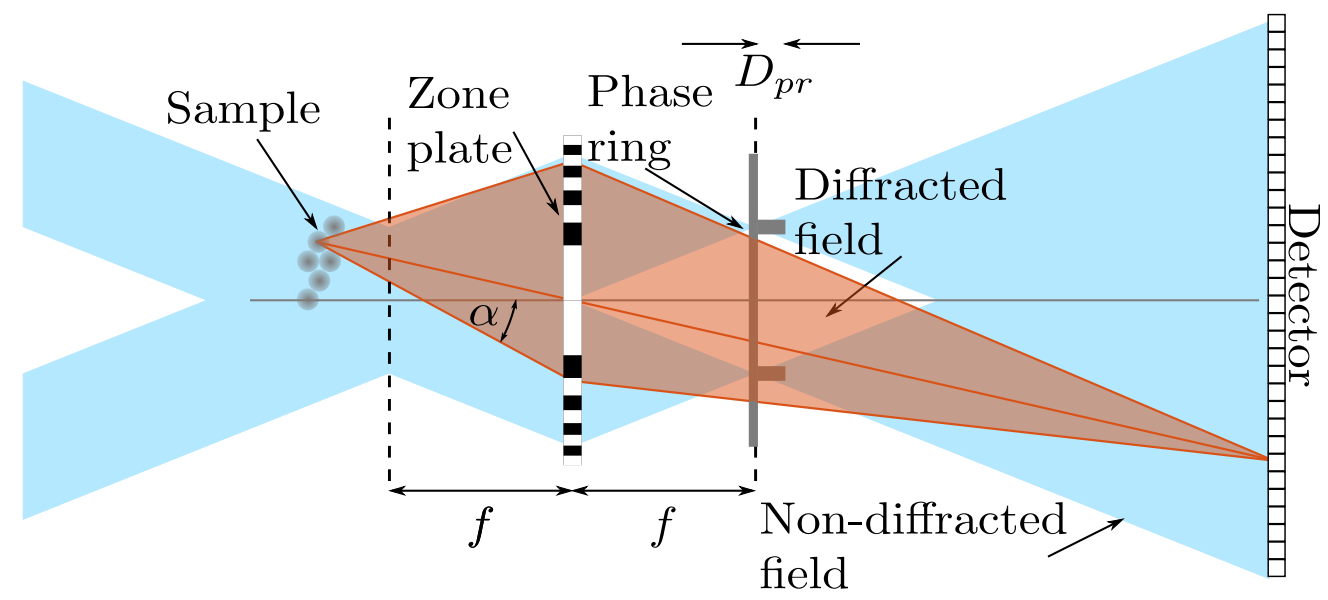

Figure 13: Schematic representation of a Zernike phase contrast X-ray microscope. Illumination must be delivered with a hollow cone by a condenser (not shown). $\alpha$ represents the maximum angle that a ray can make with the optical axis and still be focused by the zone plate and $f$ is the focal length of the zone plate. 

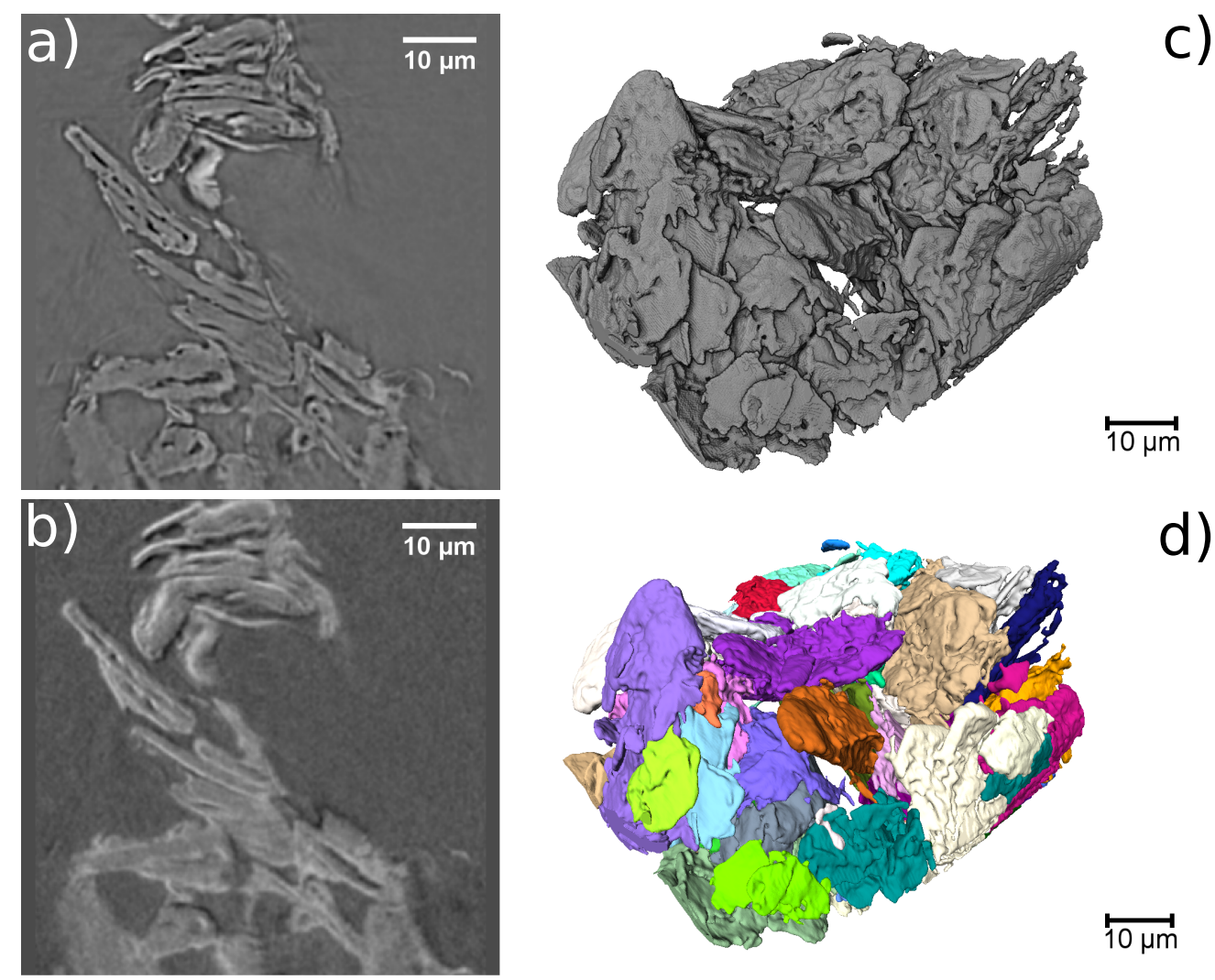

Figure 14: Images of a graphite electrode employed in a lithium-ion battery. a) Shows a single slide from the 3D phase contrast data set, b) is the same slide from the absorption data set, c) a volume rendering of the 3D combined phase and absorption contrast data set and, d) an rendering of c) but with individual graphite particles distinguished by different colors. Images contributed by O. Taiwo, adapted from data reported in [55].

a)

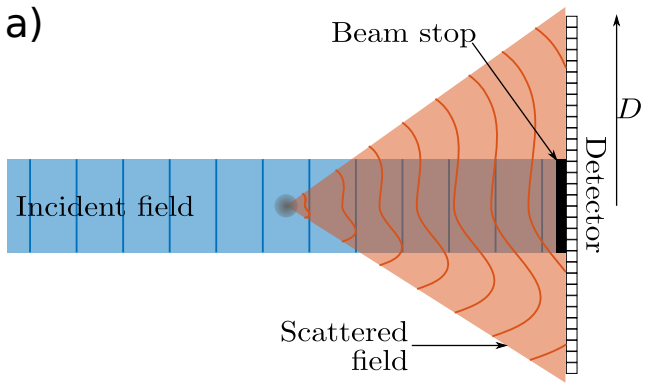

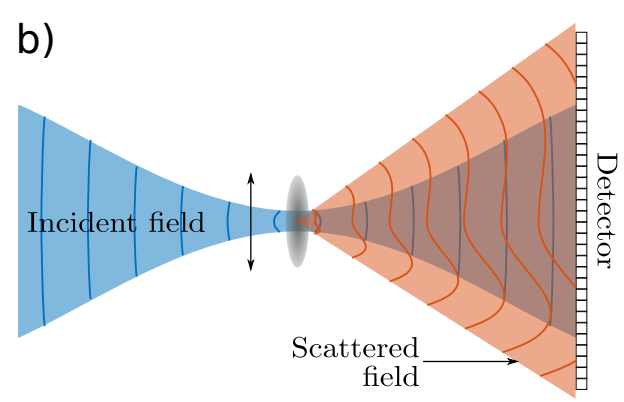

Figure 15: Schematic diagrams of configurations used to perform a) plane-wave coherent diffractive imaging, and, b) ptychography. 

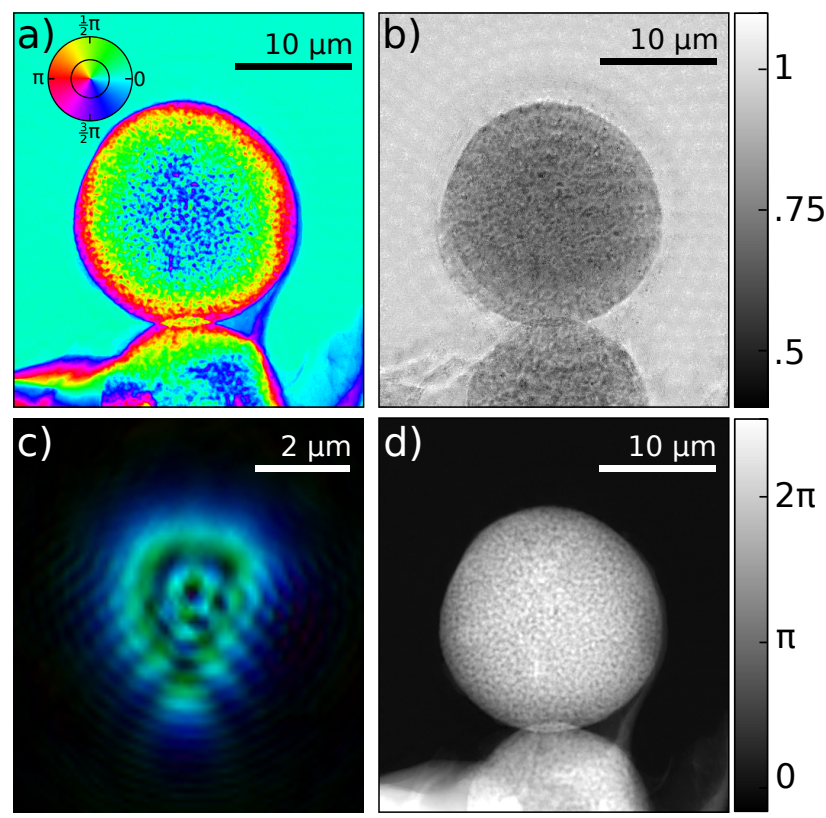

Figure 16: Example of a ptychographic reconstruction of a hydroxyapatite sphere. a) Shows the phase part (i.e., $\left.k_{0} \delta t\left(\boldsymbol{x}_{\perp}\right)\right)$, b) is the amplitude part $\left(\exp \left(-k_{0} \beta t\left(\boldsymbol{x}_{\perp}\right)\right)\right)$, c) is the reconstructed probe amplitude and $\mathrm{d}$ ) is the unwrapped phase part of the object transmission function. Image produced with the assistance and permission of Dr P. Thibault from data reported in [70]. 

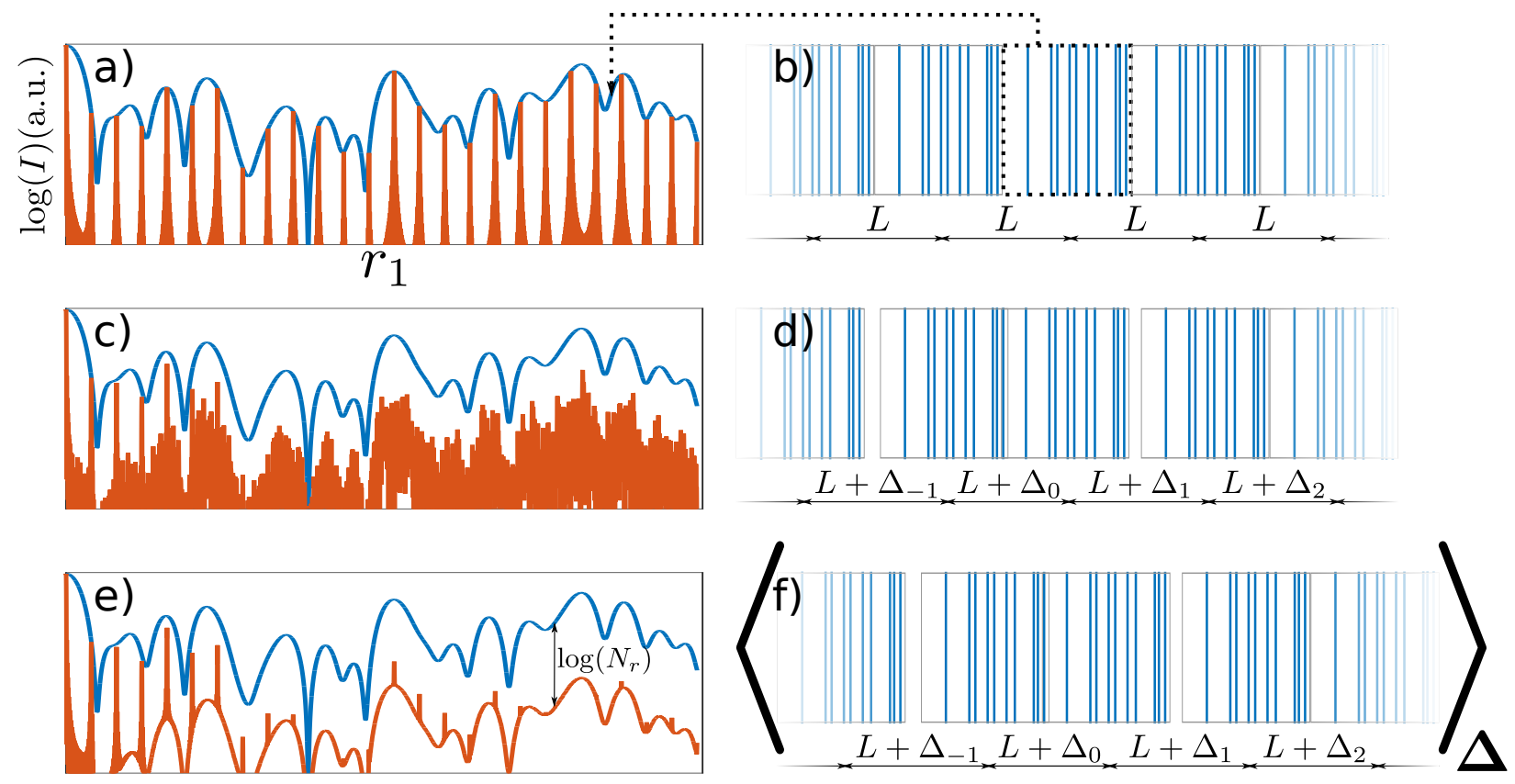

Figure 17: Schematic diagram illustrating some of the principles employed by Ayyer et. al [4] to perform macromolecular diffractive imaging. All line plots are of field intensity on a log scale covering four decades, showing only positive values of $r_{1}$ beginning at 0 . The blue plots in a), c) and e) are identical and represent the diffraction pattern due to the single aperiodic unit of apertures depicted by the broken box in b). A finite periodic extension of the aperiodic unit is shown in b) and the associated diffraction pattern plotted in red in a). The red plot in c) shows the diffraction pattern when each aperiodic unit undergoes a random shift, as shown in d). e) is obtained by averaging many realisations of the randomly shifted periodic extension, showing that away from the origin, the continuous diffraction pattern can be recovered up to a scaling factor of $N_{r}$, the number of repeated aperiodic units. All intensity plots have been normalised by the peak intensity of the continuous diffraction pattern and the number of repeated aperiodic units, in particular, the red plots have an absolute magnitude $N_{r}$ times that of the blue plots. 

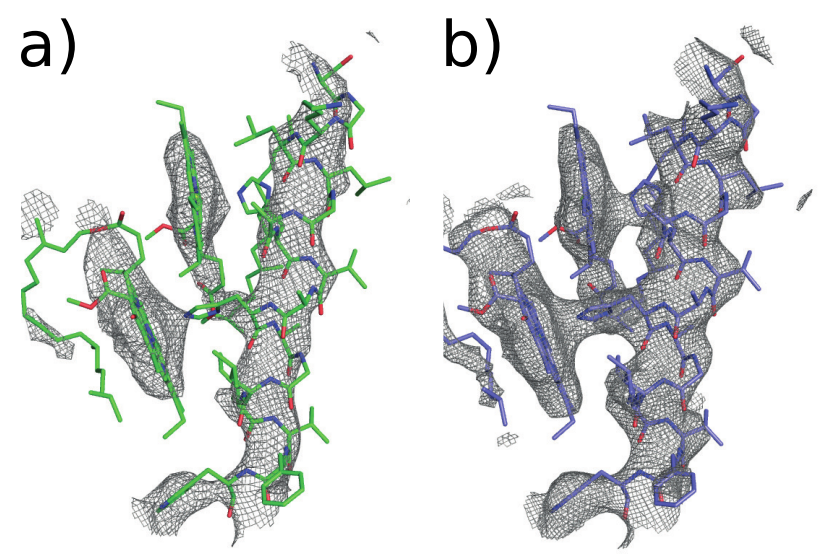

Figure 18: A region of the photosystem II membrane protein complex acquired using only Bragg information a) and at using both the Bragg and continuous diffraction data b). The mesh represents a contour at 1.5 standard deviations of the electron density. Molecular models have been derived from the electron data densities in each case. Adapted with permission from H. Chapman, and the Nature Publishing Group, from [4]. 


\section{Biography}

P.R.T. Munro completed his undergraduate study in electrical and electronic engineering at The University of Western Australia in 2000. After initially working at Telstra Research Laboratories, he began studying for his $\mathrm{PhD}$ in the Department of Physics, Imperial College London, as a Commonwealth Scholar, in 2002. He completed his PhD in optical microscopy in 2006 before completing a post-doctoral position, also at Imperial. In 2008 he took up a post-doctoral position at University College London working on the development of Xray phase imaging. He returned to The University of Western Australia in 2012 on an early career fellowship. He joined University College London in 2015 as a Royal Society University Research Fellow. His research interests are in optical microscopy, X-ray phase imaging and photoacoustic imaging and particularly theoretical aspects of image formation within these techniques.

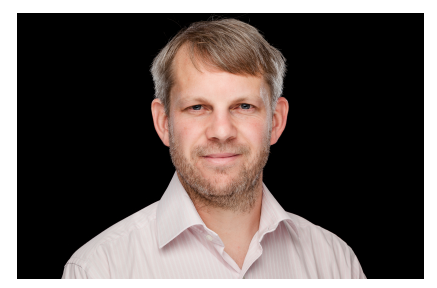

\section{References}

[1] W.C. Röntgen. On a New Kind of Rays. Nature, 53(1369):274-276, jan 1896.

[2] a Snigirev, I Snigireva, V Kohn, S Kuznetsov, and I Schelokov. On the possibilities of x-ray phase contrast imaging by coherent high-energy synchrtron radiation. Rev. Sci. Inst, 66(12):5486-5492, 1995.

[3] Peter Cloetens, Raymond Barrett, José Baruchel, Jean-Pierre Guigay, and Michel Schlenker. Phase objects in synchrotron radiation hard x-ray imaging. J. Phys. D. Appl. Phys., 29(1):133-146, jan 1996.

[4] Kartik Ayyer, Oleksandr M. Yefanov, Dominik Oberthür, Shatabdi Roy-Chowdhury, Lorenzo Galli, Valerio Mariani, Shibom Basu, Jesse Coe, Chelsie E. Conrad, Raimund Fromme, Alexander Schaffer, Katerina Dörner, Daniel James, Christopher Kupitz, Markus Metz, Garrett Nelson, Paulraj Lourdu Xavier, Kenneth R. Beyerlein, Marius Schmidt, Iosifina Sarrou, John C. H. Spence, Uwe Weierstall, Thomas A. White, JayHow Yang, Yun Zhao, Mengning Liang, Andrew Aquila, Mark S. Hunter, Joseph S. Robinson, Jason E. Koglin, Sébastien Boutet, Petra Fromme, Anton Barty, and Henry N. Chapman. Macromolecular diffractive imaging using imperfect crystals. $\mathrm{Na}$ ture, 530(7589):202-206, feb 2016. 
[5] H.M. Quiney. Coherent diffractive imaging using short wavelength light sources. J. Mod. Opt., 57(13):1109-1149, 2010.

[6] K. Nugent. Coherent methods in the x-ray sciences. Adv. Phys., 59(1):1-99, 2010.

[7] Henry N. Chapman and Keith a. Nugent. Coherent lensless X-ray imaging. Nat. Photonics, 4(12):833-839, 2010.

[8] K.A. Nugent. The measurement of phase through the propagation of intensity: an introduction. Contemporary Physics, 52(1):55-69, 2011.

[9] Roger Falcone, Chris Jacobsen, Janos Kirz, Stefano Marchesini, David Shapiro, and John Spence. New directions in X-ray microscopy. Contemp. Phys., 52(4):293-318, 2011.

[10] J C H Spence, U Weierstall, and H N Chapman. X-ray lasers for structural and dynamic biology. Reports Prog. Phys., 75(10):102601, 2012.

[11] Kerstin Galler, Katharina Bräutigam, Christina Große, Jürgen Popp, and Ute Neugebauer. Making a big thing of a small cell recent advances in single cell analysis. Analyst, 139(6):1237, 2014.

[12] Jianwei Miao, Tetsuya Ishikawa, Ian K Robinson, and Margaret M Murnane. Beyond crystallography: Diffractive imaging using coherent x-ray light sources. Science, 348(6234):530-535, 2015.

[13] S W Wilkins, Ya I Nesterets, T E Gureyev, S. C. Mayo, A. Pogany, and A. W. Stevenson. On the evolution and relative merits of hard X-ray phase-contrast imaging methods. Philos. Trans. R. Soc. A Math. Phys. Eng. Sci., 372(2010):20130021-20130021, jan 2014.

[14] J.D. Jackson. Classical Electrodynamics. John Wiley \& Sons, New York, third edition, 1999.

[15] D. Paganin. Coherent X-ray optics. Oxford series on synchrotron radiation. Oxford University Press, Great Clarendon Street, Oxford OX2 6DP, 2006.

[16] M. Born and E. Wolf. Principles of Optics. Cambridge University Press, Cambridge, seventh edition, 1999.

[17] B.L. Henke, E.M. Gullikson, and J.C. Davis. X-ray interactions: Photoabsorption, scattering, transmission, and reflection at $\mathrm{e}=50-30,000 \mathrm{ev}, \mathrm{z}=1-92$. Atom. Data Nucl. Data, 54(2):181 - 342, 1993.

[18] J.W. Goodman. Introduction to Fourier Optics. McGraw-Hill, New York, 1988. 
[19] Kaye S. Morgan, Karen K W Siu, and David M. Paganin. The projection approximation versus an exact solution for X-ray phase contrast imaging, with a plane wave scattered by a dielectric cylinder. Opt. Commun., 283(23):4601-4608, 2010.

[20] E. Wolf. Introduction to the theory of coherence and polarization of light. Cambridge University Press, 2007.

[21] L. Mandel and E. Wolf. Optical Coherence and Quantum Optics. Cambridge University Press, Cambridge, 1995.

[22] Pratik Shukla, Jonathan Lawrence, and Yu Zhang. Understanding laser beam brightness: A review and new prospective in material processing. Opt. Laser Technol., 75:40$51,2015$.

[23] Thorsten M Buzug. Computed Tomography. Springer Berlin Heidelberg, Berlin, Heidelberg, 2008.

[24] D. T. Attwood and K. J. Kim. Spectral brightness and coherent power of radiation from high brightness 1-6 GeV storage rings. Nucl. Inst. Methods Phys. Res. A, 246(13):86-90, 1986.

[25] Steven L. Hulbert and Jill M. Weber. Flux and brightness calculations for various synchrotron radiation sources. Nucl. Inst. Methods Phys. Res. A, 319(1-3):25-31, 1992.

[26] Margaret M. Martin. Daresbury SRS. Synchrotron Radiat. News, 1(3):14-18, may 1988.

[27] V.P Suller, J.N. Corlett, D.M. Dykes, E.A. Hughes, M.W. Poole, P.D. Quinn, J.S. MacKay, S.L. Thompson, and R.P. Walker. Performance of the Daresbury SRS with an increased brilliance optic. In Eur. Part. Accel. Conf., 1988.

[28] P.R.T Munro, K. Ignatyev, R.D. Speller, and A. Olivo. The relationship between wave and geometrical optics models of coded aperture type x-ray phase contrast imaging systems. Opt. Express, 18(5):4103-4117, 2010.

[29] Samuel Flewett, Harry M. Quiney, Chanh Q. Tran, and Keith A. Nugent. Extracting coherent modes from partially coherent wavefields. OPTICS LETTERS, 34(14):21982200, JUL 152009.

[30] D. Dreossi, A. Abrami, F. Arfelli, R. Bregant, K. Casarin, V. Chenda, M. A. Cova, R. Longo, R. H. Menk, E. Quai, E. Quaia, L. Rigon, T. Rokvic, D. Sanabor, M. Tonutti, G. Tromba, A. Vascotto, F. Zanconati, and E. Castelli. The mammography project at the syrmep beamline. European Journal of Radiology, 68(3):S58-S62, DEC 2008. Conference on Medical Applications of Synchrotron Radiation, Saskatoon, CANADA, 2007. 
[31] M. J. Kitchen, R. a. Lewis, N. Yagi, K. Uesugi, D. Paganin, S. B. Hooper, G. Adams, S. Jureczek, J. Singh, C. R. Christensen, a. P. Hufton, C. J. Hall, K. C. Cheung, and K. M. Pavlov. Phase contrast X-ray imaging of mice and rabbit lungs: A comparative study. Br. J. Radiol., 78(935):1018-1027, 2005.

[32] Mark W Westneat. Tracheal Respiration in Insects Visualized with Synchrotron X-ray Imaging. Science, 299(5606):558-560, jan 2003.

[33] E Quai, R Longo, F Zanconati, G Jaconelli, M Tonutti, A Abrami, F Arfelli, D Dreossi, $\mathrm{G}$ Tromba, and $\mathrm{M}$ a Cova. First application of computed radiology to mammography with synchrotron radiation. Radiol. Med., 118(1):89-100, feb 2013.

[34] E Castelli, M Tonutti, F Arfelli, R Longo, E Quaia, L Rigon, D Sanabor, F Zanconati, D Dreossi, A Abrami, E Quai, P Bregant, K Casarin, V Chenda, R H Menk, T Rokvic, A Vascotto, G Tromba, and M a Cova. Mammography with Synchrotron Radiation: First Clinical Experience with Phase-Detection Technique. Radiology, 259(3):684-694, 2011.

[35] Renata Longo, Maura Tonutti, Luigi Rigon, Fulvia Arfelli, Diego Dreossi, Elisa Quai, Fabrizio Zanconati, Edoardo Castelli, Giuliana Tromba, and Maria A Cova. Clinical study in phase- contrast mammography: image-quality analysis. Philos. Trans. R. Soc. London A Math. Phys. Eng. Sci., 372(2010):1-8, 2014.

[36] R. A. Lewis, C. J. Hall, A. P. Hufton, S. Evans, R. H. Menk, F. Arfell, L. Rigon, G. Tromba, D. R. Dance, O. Ellis, A. Evans, E. Jacobs, S. E. Pinder, and K. D. Rogers. X-ray refraction effects: Application to the imaging of biological tissues. Br. J. Radiol., 76(905):301-308, 2003.

[37] O. Wernick, M.N. Wirjadi, D. Chapman, Z. Zhong, N.P. Galatsanos, Y. Yang, J.G. Brankov, O. Oltulu, M.A. Anastasio, and C. Muehleman. Multiple-image radiography. Physics in Medicine and Biology, 48(23):38753895, 2003.

[38] E Pagot, P Cloetens, S Fiedler, A Bravin, P Coan, J Baruchel, J Hartwig, and W Thomlinson. A method to extract quantitative information in analyzer-based x-ray phase contrast imaging. Appl. Phys. Lett., 82(20):3421-3423, MAY 192003.

[39] K. Patorski. The self-imaging phenomenon and its applications. Prog. Optics, XXVII:1108, 1989.

[40] T. Weitkamp, A. Diaz, C. David, F. Pfeiffer, M. Stampanoni, P. Cloetens, and E. Ziegler. X-ray phase imaging with a grating interferometer. Opt. Express, 13(16):6296-6304, 2005.

[41] A. Momose, W. Yashiro, Y. Takeda, Y. Suzuki, and T. Hattori. Phase tomography by x-ray talbot interferometry for biological imaging. Jpn. J. Appl. Phys., 45:5254-5262, 2006. 
[42] P.R.T Munro, K. Ignatyev, R.D. Speller, and A. Olivo. Phase and absorption retrieval using incoherent x-ray sources. Proc. Natl. Acad. Sci. U. S. A., 109:13922-13927, 2012.

[43] P.R.T Munro, C.K. Hagen, M.B. Szafraniec, and A. Olivo. A simplified approach to quantitative coded aperture x-ray phase imaging. Opt. Express, 21:11187-11201, 2013.

[44] Paul C. Diemoz, Fabio a. Vittoria, Charlotte K. Hagen, Marco Endrizzi, Paola Coan, Emmanuel Brun, Ulrich H. Wagner, Christoph Rau, Ian K. Robinson, Alberto Bravin, and Alessandro Olivo. Single-image phase retrieval using an edge illumination X-ray phase-contrast imaging setup. J. Synchrotron Radiat., 22(4):1072-1077, 2015.

[45] Peter R.T. Munro, Luigi Rigon, Konstantin Ignatyev, Frances C.M. Lopez, Diego Dreossi, Robert D. Speller, and Alessandro Olivo21:647-661. A quantitative, noninterferometric x-ray phase contrast imaging technique. Opt. Express, 21(1):647-661, Jan 2013.

[46] A. Snigirev, V. Kohn, I. Snigireva, and B. Lengeler. A compound refractive lens for focusing high-energy X-rays. Nature, 384(6604):49-51, 1996.

[47] C. Schroer and B. Lengeler. Focusing Hard X Rays to Nanometer Dimensions by Adiabatically Focusing Lenses. Phys. Rev. Lett., 94(5):054802, 2005.

[48] Albert V Baez. Fresnel Zone Plate for Optical Image Formation Using Extreme Ultraviolet and Soft X Radiation. J. Opt. Soc. Am., 51(4):405-412, 1961.

[49] B. Niemann, D. Rudolph, and G. Schmahl. X-ray microscopy with synchrotron radiation. Appl. Opt., 15(8):1883, aug 1976.

[50] J.M. Kenney, J. Kirz, H. Rarback, M.R. Howells, P. Chang, P.J. Coane, R. Feder, P.J. Houzego, D.P. Kern, and D. Sayre. Soft X-ray microscopy at the NSLS. Nucl. Instruments Methods Phys. Res., 222(1-2):37-41, may 1984.

[51] F. Zernike. How i discovered phase contrast. Science, 121:345-349, 1955.

[52] G Schmahl, D Rudolph, G Schneider, P Guttmann, and B Niemann. schmahl94optik181.pdf. Optik (Stuttg)., 97(4):181-182, 1994.

[53] Marco Stampanoni, Rajmund Mokso, Federica Marone, Joan Vila-Comamala, Sergey Gorelick, Pavel Trtik, Konstantin Jefimovs, and Christian David. Phase-contrast tomography at the nanoscale using hard x rays. Phys. Rev. B - Condens. Matter Mater. Phys., 81(14):1-4, 2010.

[54] Ismo Vartiainen, Martin Warmer, Dennis Goeries, Eva Herker, Rudolph Reimer, Christian David, and Alke Meents. Towards tender X-rays with Zernike phase-contrast imaging of biological samples at $50 \mathrm{~nm}$ resolution. J. Synchrotron Radiat., 21(4):790-794, 2014. 
[55] Oluwadamilola O. Taiwo, Donal P. Finegan, Jeff Gelb, Christian Holzner, Daniel J.L. Brett, and Paul R. Shearing. The use of contrast enhancement techniques in X-ray imaging of lithiumion battery electrodes. Chem. Eng. Sci., apr 2016.

[56] R W Gerchberg and W O Saxton. A practical algorithm for the determination of phase from image and diffraction plane pictures. Optik (Stuttg)., 35(2):237-246, 1972.

[57] J R Fienup. Phase retrieval algorithms: a personal tour [Invited]. Appl. Opt., 52(1):4556, 2012.

[58] J R Fienup. Phase retrieval algorithms: a comparison. Appl. Opt., 21(15):2758-2769, 1982.

[59] WR Fright and RHT Bates. fright82Optik219.pdf. Optik (Stuttg)., 62(3):219-230, 1982.

[60] LH Garden and RHT Bates. garden82Optik131.pdf. Optik (Stuttg)., 62(2):131-142, 1982.

[61] RHT Bates. Fourier phase problems are uniquely solvable in more than one dimension, 1. Underlying theory, 1981.

[62] J Miao, D Sayre, and H N Chapman. Phase retrieval from the magnitude of the Fourier transforms of nonperiodic objects. J. Opt. Soc. Am. A, 15(6):1662, 1997.

[63] Jianwei Miao, Pambos Charalambous, Janos Kirz, and David Sayre. Extending the methodology of X-ray crystallography to allow imaging of micrometre-sized noncrystalline specimens. Nature, 400(6742):342-344, 1999.

[64] J Miao, K O Hodgson, and D Sayre. An approach to three-dimensional structures of biomolecules by using single-molecule diffraction images. Proc. Natl. Acad. Sci. U. S. A., 98(12):6641-6645, 2001.

[65] Henry N. Chapman, Anton Barty, Stefano Marchesini, Aleksandr Noy, Stefan P. HauRiege, Congwu Cui, Malcolm R. Howells, Rachel Rosen, Haifeng He, John C. H. Spence, Uwe Weierstall, Tobias Beetz, Chris Jacobsen, and David Shapiro. High-resolution ab initio three-dimensional x-ray diffraction microscopy. J. Opt. Soc. Am. A, 23(5):1179, 2006.

[66] Brian Abbey, Keith A Nugent, Garth J Williams, Jesse N Clark, Andrew G Peele, Mark A Pfeifer, Martin de Jonge, and Ian McNulty. Keyhole coherent diffractive imaging. Nat. Phys., 4(5):394-398, 2008.

[67] P. Thibault, M. Dierolf, A. Menzel, O. Bunk, C. David, and F. Pfeiffer. High-Resolution Scanning X-ray Diffraction Microscopy. Science, 321(5887):379-382, 2008.

[68] J. M. Rodenburg and R. H. T. Bates. The Theory of Super-Resolution Electron Microscopy Via Wigner-Distribution Deconvolution. Philos. Trans. R. Soc. A Math. Phys. Eng. Sci., 339(1655):521-553, jun 1992. 
[69] J. M. Rodenburg, A. C. Hurst, A. G. Cullis, B. R. Dobson, F. Pfeiffer, O. Bunk, C. David, K. Jefimovs, and I. Johnson. Hard-x-ray lensless imaging of extended objects. Phys. Rev. Lett., 98(3), JAN 192007.

[70] P. Thibault and M. Guizar-Sicairos. Maximum-likelihood refinement for coherent diffractive imaging. New J. Phys., 14:0-20, 2012.

[71] M. R. Howells, T. Beetz, H. N. Chapman, C. Cui, J. M. Holton, C. J. Jacobsen, J. Kirz, E. Lima, S. Marchesini, H. Miao, D. Sayre, D. A. Shapiro, J. C H Spence, and D. Starodub. An assessment of the resolution limitation due to radiation-damage in X-ray diffraction microscopy. J. Electron Spectros. Relat. Phenomena, 170(1-3):4-12, 2009.

[72] R Neutze, R Wouts, D van der Spoel, E Weckert, and J Hajdu. Potential for biomolecular imaging with femtosecond X-ray pulses. Nature, 406(6797):752-757, 2000.

[73] N W Ashcroft and N D Mermin. Solid state physics. Thomson Learning, 1976.

[74] C Hammond. The basics of crystallography and diffraction. Oxford University Press, Oxford, 2001.

[75] Richard A Kirian, Xiaoyu Wang, Uwe Weierstall, Kevin E Schmidt, John C H Spence, Mark Hunter, Petra Fromme, Thomas White, Henry N Chapman, and James Holton. Femtosecond protein nanocrystallographydata analysis methods. Opt. Express, 18(6):5713-5723, 2010.

[76] P. Emma, R. Akre, J. Arthur, R. Bionta, C. Bostedt, J. Bozek, A. Brachmann, P. Bucksbaum, R. Coffee, F.-J. Decker, Y. Ding, D. Dowell, S. Edstrom, A. Fisher, J. Frisch, S. Gilevich, J. Hastings, G. Hays, Ph. Hering, Z. Huang, R. Iverson, H. Loos, M. Messerschmidt, A. Miahnahri, S. Moeller, H.-D. Nuhn, G. Pile, D. Ratner, J. Rzepiela, D. Schultz, T. Smith, P. Stefan, H. Tompkins, J. Turner, J. Welch, W. White, J. Wu, G. Yocky, and J. Galayda. First lasing and operation of an ångstrom-wavelength freeelectron laser. Nat. Photonics, 4(9):641-647, 2010.

[77] Gernot Renger. Mechanism of light induced water splitting in Photosystem II of oxygen evolving photosynthetic organisms. Biochim. Biophys. Acta - Bioenerg., 1817(8):1164$1176,2012$. 